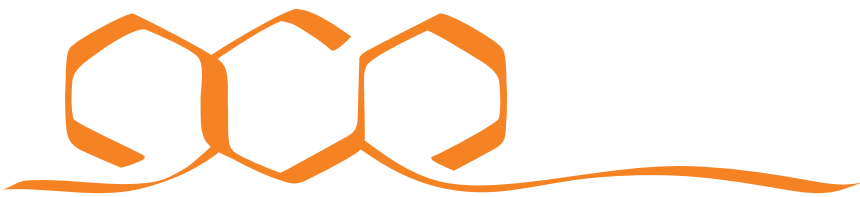 \\ COMMUNICATIONS CHEMISTRY
}

REVIEW ARTICLE

https://doi.org/10.1038/s42004-018-0098-8

OPEN

\section{Approaches and challenges in the synthesis of three-dimensional covalent-organic frameworks}

\author{
Xingjian Ma (1D ${ }^{1}$ \& Timothy F. Scott (10 ${ }^{1,2}$
}

Covalent organic frameworks, cross-linked crystalline polymers constructed from rigid organic precursors connected by covalent interactions, have emerged as a promising class of nanoporous materials owing to their highly desirable combination of attributes, including facile chemical tunability, structural diversity, and excellent stability. Despite the distinct advantages offered by three-dimensional covalent organic frameworks, research efforts have predominantly focused on the more synthetically-accessible, two-dimensional variants. Here we present an overview of synthetic approaches to yield three-dimensional covalent organic frameworks, identify synthetic obstacles that have hindered progress in the field and recently-employed methods to address them, and propose alternative techniques to circumvent these synthetic challenges.

$\mathrm{N}$ anoporous materials have garnered tremendous interest in recent years owing to their specific and exceptional attributes, notably permanent porosity and large and accessible internal surface areas ${ }^{1-5}$. Conventional nanoporous materials used commonly as adsorbents and heterogeneous catalysts and catalyst supports, such as zeolites and activated carbon, are based on inorganic building blocks; nevertheless, research interest in nanoporous materials bearing organic components has expanded rapidly ${ }^{1,5,6}$. This is in part due to the exquisite structural and functional control such components provide and the flexibility they afford for designing materials specifically tailored towards the intended application ${ }^{5}$.

A large number of such nanoporous organic polymers have been fabricated in recent years, including polymers of intrinsic microporosity (PIMs) ${ }^{5,6}$, porous polymer networks (PPNs) ${ }^{7}$, and conjugated microporous polymers (CMPs) ${ }^{8-10}$. These materials are uniformly amorphous and composed of multifunctional building blocks linked by covalent bonds, and many studies have demonstrated their synthesis and utility $7,11-13$. Unfortunately, the amorphous nature of these materials can yield significant pore size dispersities, inhibiting their utilization in certain applications such as size- and shape-based gas separation and storage ${ }^{2,11}$.

Crystallinity, in contrast, is characterized by ordered structure and uniform porosity, ideal attributes for gas separation and storage, catalysis, and optoelectronic devices ${ }^{2,14}$. Metal-organic frameworks (MOFs) are one such class of crystalline nanoporous materials that have been investigated extensively. MOFs are constructed from metal ions or clusters linked by organic

\footnotetext{
${ }^{1}$ Department of Chemical Engineering, University of Michigan, Ann Arbor, MI 48109, USA. ${ }^{2}$ Macromolecular Science and Engineering Program, University of Michigan, Ann Arbor, Ml 48109, USA. Correspondence and requests for materials should be addressed to T.F.S. (email: tfscott@umich.edu)
} 
ligands through coordination bonds, thus possessing both the chemical and structural tunability of the organic ligands, the coordination possibilities of various metal ions, and the uniform porosity and high surface area of a crystalline nanoporous material $^{3,15-17}$. Examples of this class of materials necessarily contain a large amount of potentially toxic or reactive metal centers, and their relatively weak coordination bonds exhibit varying stability under high humidity and temperature, conditions ubiquitous in industrial applications ${ }^{18-21}$.

A particularly exciting development in this field was the landmark synthesis of covalent-organic frameworks (COFs) by Yaghi and coworkers in $2005^{22}$. In this seminal work, two COFs were synthesized from the self-condensation of 1,4-benzenediboronic acid (COF-1) or co-condensation with hexahydroxytriphenylene (COF-5), establishing the first class of crystalline nanoporous organic frameworks linked exclusively by covalent bonds. Although the strength of covalent bonds contributes to significantly improved stability, the general irreversibility of kinetically controlled reactions prevents the molecular rearrangement necessary to form a crystalline structure. Fortunately, the addition products of several covalent bond-forming reactions are known to either revert to the constituent reactants or rearrange under specific reaction conditions. These dynamic covalent reactions, utilizing reversible covalent bonds, which can be formed and broken rapidly under appropriate conditions, are capable of obtaining thermodynamically as opposed to kinetically controlled product distributions. This thereby provides a mechanism for error-correction and facilitates the assembly of the most thermodynamically stable covalent structure, equivalent to conventional self-assembly with non-covalent, intermolecular interactions ${ }^{19}$. In contrast with the aforementioned amorphous nanoporous polymers and MOFs, COFs offer a unique combination of chemical modularity and structural diversity, crystallinity, high surface area, tunable pore size, thermal stability, and low density $3,11,14,23$.

Based on the dimensionality of the covalent connectivity, COFs can be classified as either two-dimensional (2D) COFs or threedimensional (3D) COFs. Two-dimensional COFs are fabricated from planar building blocks, and the framework is restricted to $2 \mathrm{D}$ sheets, which can then be stacked to form a layered eclipsed structure through, e.g., $\pi$-orbital overlap, generating ordered, onedimensional channels that are well-suited for charge transport ${ }^{1,3}$. In contrast, 3D COFs are synthesized from aplanar (typically tetrahedral) building blocks to form highly porous networks, which are similar in structure to MOFs and have been found to possess exceptionally high surface areas $\left(>5000 \mathrm{~m}^{2} / \mathrm{g}\right)$ and low densities $\left(<0.13 \mathrm{~g} / \mathrm{cm}^{3}\right)^{24}$, making them attractive synthetic targets. Indeed, although studies examining 3D COF applications remain sparse, several simulation and experimental studies have assessed the gas storage capacity of $3 \mathrm{D}$ COFs and predicted or empirically demonstrated performance equal to or exceeding the best-performing MOFs owing to their exceptional porosity, surface area, and low density ${ }^{25-30}$. In addition, the promising performance of $3 \mathrm{D}$ COFs as superb adsorbents has also been shown ${ }^{31}$.

Despite the attractive attributes of 3D COFs, research efforts have predominantly focused on their more synthetically accessible 2D counterparts. This review seeks to provide a comprehensive overview of the current state of $3 \mathrm{D}$ COFs, address the synthetic challenges attributable to the significant disparity between $2 \mathrm{D}$ and $3 \mathrm{D}$ COFs, and submit alternative approaches that might be employed to circumvent these synthetic challenges.

\section{Linkages and modification of 3D COFs}

Overview. The first synthesis of 3D COFs, reported in 2007 again by Yaghi and coworkers, brought a new dimension to the field ${ }^{32}$. There have since been many reports describing new 2D COF structures ${ }^{33,34}$, novel synthetic strategies such as microwave synthesis $^{35}$, flow synthesis ${ }^{36-38}$, and vapor-assisted synthesis ${ }^{39,40}$, and construction and modification of 2D COFs towards applications including catalysis ${ }^{41,42}$, membrane fabrication ${ }^{43}$, and gas storage $^{44}$. In comparison, there have been only a handful of new 3D COF structures reported, and little beyond structural explorations and preliminary applications. Whereas a variety of dynamic covalent bond-forming reactions have been employed for the synthesis of $2 \mathrm{D}$ COFs, only a few have been used for constructing $3 \mathrm{D}$ structures. Consequently, the linkages that govern 3D COFs can be generally categorized as boron-oxygen linkages, imine linkages, and other linkage types. A list of linkage chemistries that have been reported to date for $3 \mathrm{D}$ COFs is shown in Fig. 1.

Boron-oxygen linkages. Most early 3D COF structures were formed utilizing boron-oxygen (B-O) linkages. The first 3D COFs, COF-102, COF-103, COF-105, and COF-108 were synthesized from the self-condensation of boronic acids into boroxine rings and co-condensation with catechol into boronate ester rings (Fig. 2a, b). These syntheses were performed under solvothermal conditions from suspensions of precursors in a mixture of mesitylene/dioxane, resulting in the lowest density crystals known at the time (COF-108, $\left.0.17 \mathrm{~g} / \mathrm{cm}^{3}\right)$ and BrunauerEmmett-Teller (BET) surface areas (COF-103, $4210 \mathrm{~m}^{2} / \mathrm{g}$ ) far exceeding those of porous carbons, silicates, 2D COFs, and PIMs, and comparable to some of the highest reported for MOFs (MOF$\left.177,4500 \mathrm{~m}^{2} / \mathrm{g}\right)^{32}$. Although rapid microwave synthesis and purification was later successfully demonstrated for both $2 \mathrm{D}$ and $3 \mathrm{D}$ boroxine and boronate ester-linked COFs, this approach has not received significant further study for $3 \mathrm{D}$ COFs, and the solvothermal approach has emerged as the prevalent method for $3 \mathrm{D}$ COF synthesis ${ }^{32,45}$. In addition to boroxine and boronate ester linkages, a borosilicate cage was also utilized as a linkage for the synthesis of COF-202 (Fig. 2c) ${ }^{46}$.

The exceptionally low densities and high-surface areas of these 3D COFs makes them highly promising gas storage candidates. Indeed, grand canonical Monte Carlo (GCMC) simulations on $\mathrm{H}_{2}$ binding for $2 \mathrm{D}$ and $3 \mathrm{D}$ COFs containing $\mathrm{B}-\mathrm{O}$ linkages, which was confirmed experimentally for COF-105, predicted that 3D COFs outperform 2D COFs in hydrogen storage capacity by a factor of 2.5-3, attributable to higher surface area and free volume in the $3 \mathrm{D}$ variants $^{26}$. Three-dimensional COFs were also expected to significantly outperform the best-performing MOFs in $\mathrm{H}_{2}$ gravimetric uptake owing to reduced densities and comparable volumetric storage capacity, while doping with lithium ions was anticipated to further increase storage capacity ${ }^{26-28}$. Subsequent experimental investigation of the $\mathrm{H}_{2}$ storage capacity of these COFs confirmed that these $3 \mathrm{D}$ COFs demonstrate best-in-class $\mathrm{H}_{2}$ saturation uptake (72.4 mg/g for COF-102), far exceeding 2D COFs $(39.2 \mathrm{mg} / \mathrm{g}$ for COF-10) and comparable to MOFs (72.4 $\mathrm{mg} / \mathrm{g}$ for MOF-5) at $77 \mathrm{~K}$. The same study also showed that $3 \mathrm{D}$ COFs perform remarkably in the storage of methane and carbon dioxide, comparing favorably to other nanoporous materials including zeolites and MOFs, and that larger intrinsic surface area (or pore volume) generally correlate to higher uptake capacity for these gases ${ }^{30}$. Further theoretical studies predicted that COF-102 and COF-103 outperform other $2 \mathrm{D}$ and $3 \mathrm{D}$ COFs and the benchmark MOF-177 in volumetric uptake and delivery amount of methane at high pressure ${ }^{25}$; however, 3D COFs were predicted to exhibit only average hydrogen storage capacity compared to other MOFs, and required the introduction of stronger $\mathrm{H}_{2}$ interaction sites in the COF material for improved performance 29,47 . In addition to small gas molecules, the organometallic host-guest chemistry of COF-102 was also examined by infiltration of metallocenes, and similarities between MOFs and COFs as hosts for organometallics was demonstrated ${ }^{48}$. 


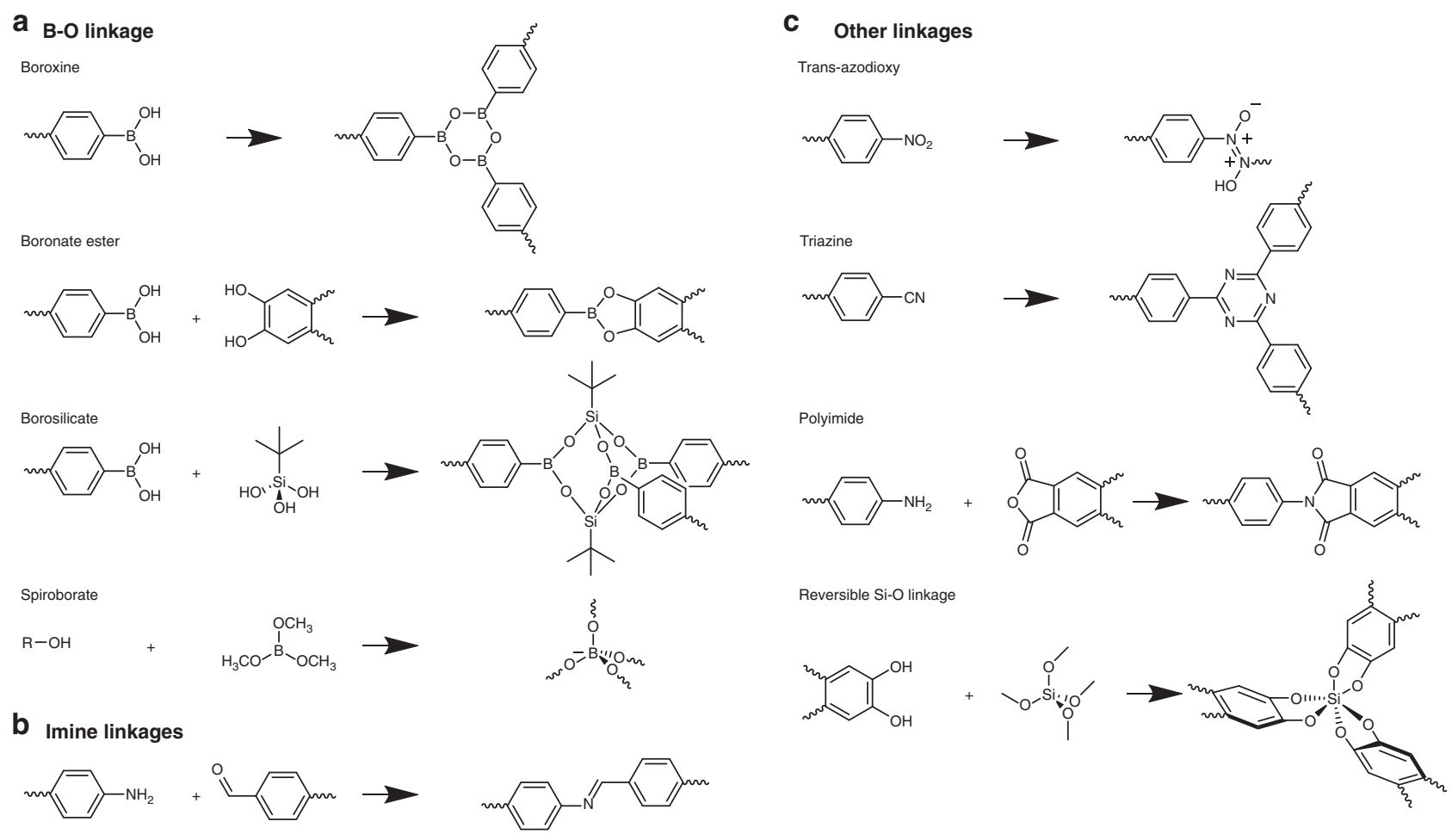

Fig. 1 Reported synthetic approaches and accompanying linkages used to generate 3D COFs. a Reported B-O linkages, including boroxine ring, boronate ester ring, borosilicate cage, and spiroborate linkage. b The imine linkage. c Other reported linkages, including trans-azodioxy linkage, triazine linkage, polyimide linkage, and reversible Si-O linkage

More recently, metalation of a 3D COF containing B-O linkage was also demonstrated utilizing dehydrobenzoannulene (DBA), a planar triangular macrocycle that can complex with low oxidation state transition metals, as a building block. The synthesized DBA3D-COF-1 (Fig. 2d) possesses the highest BET surface area (5083 $\left.\mathrm{m}^{2} / \mathrm{g}\right)$ and lowest density $\left(0.13 \mathrm{~g} / \mathrm{cm}^{3}\right)$ reported to date for a COF, and subsequent metalation of the COF with nickel resulted in retention of crystallinity and minimal reduction in surface area and pore volume ${ }^{24}$. In addition, a 3D, $\gamma$-cyclodextrin-based COF (CD-COF) was synthesized utilizing a unique tetrakis(spiroborate) linkage, marking the first instance of a 3D anionic COF constructed from flexible aliphatic building blocks (Fig. 3a ${ }^{49}$.

Imine linkages. Although many of the early 3D COFs relied on $\mathrm{B}-\mathrm{O}$ linkages, these linkages suffer from poor stability towards hydrolysis and oxidation, limiting their practical utility. Consequently there has been a notable trend away from such linkages towards more robust nitrogen-containing linkage chemistries for $\mathrm{COF}$ design and synthesis ${ }^{50}$. Imine linkages, synthesized from multi-functional, amine- and aldehyde-bearing precursors, currently dominate the field of 3D COFs owing to their excellent thermal and chemical stability.

The first imine-linked 3D COF, COF-300, was reported by Yaghi and coworkers in 2009, and is synthesized from tetrakis-(4aminophenyl)methane (TAPM) and terephthalaldehyde (Fig. 4b). COF-300 was assigned as a fivefold interpenetrated diamond structure (dia-c5 topology), is stable up to $490^{\circ} \mathrm{C}$, and is insoluble in water and common organic solvents ${ }^{51}$. It was later reported that by first aging the reaction mixture prior to synthesis, an interpenetration isomer dia-c7 COF-300 possessing a sevenfold interpenetrated diamond structure, could be obtained ${ }^{52}$. The two isomers exhibited identical covalent bonding at the atomic level and identical thermal stability but were structurally different as revealed by X-ray diffractometry. Ma et al. ${ }^{53}$ recently reported the construction of single-crystal COF-300 by employing a large excess of aniline as a nucleation inhibitor to prevent immediate precipitation of amorphous networks and increase the reversibility of imine bond formation and dissociation ${ }^{53}$. They showed that crystal size could be controlled by adjusting aniline concentration and were able to obtain single COF-300 crystals of up to $100 \mu \mathrm{m}$ in size after 30-40 days.

Single-crystal X-ray diffraction (SCXRD) characterization allowed the resolution of ambiguity in the interpenetration and revealed that COF-300 exhibits a sevenfold interpenetrated structure, and that previous assessment of fivefold interpenetration was owing to structural contortion. SCXRD measurements also revealed that hydration of COF-300 led to structural distortion and a drastic 34\% reduction in unit-cell volume, which may be attributed to hydrogen bonding between water molecules and the imine bonds. The authors also synthesized COF-303, an analog of COF-300 with a reversed imine condensation between a tetratopic aldehyde and diamine, and demonstrated that the switch in imine bond direction was not differentiable by conventional powder X-ray diffraction (PXRD), but could be detected by SCXRD ${ }^{53}$.

COF-300 has also been utilized in conjunction with the zirconium-carboxylate-based MOF UiO-66 to fabricate a composite laminate where a COF-300 layer was grown on top of a prefabricated UiO-66 membrane. These composite membranes demonstrated excellent $\mathrm{H}_{2}$ permeability as well as a highly enhanced $\mathrm{H}_{2} / \mathrm{CO}_{2}$ selectivity, far surpassing that of the parent UiO-66 membrane ${ }^{54}$. Additionally, layer-by-layer synthesis of COF-300/silica composites was recently demonstrated to afford particles with potential utility as a chromatographic stationary phase ${ }^{55}$. Yaghi and coworkers also reported COF-320, essentially an extended version of COF-300 constructed from a biphenyl dialdehyde linker, and determined its single-crystal structure using $3 \mathrm{D}$ rotation electron diffraction (Fig. $4 \mathrm{c})^{56}$. COF-320 was 
a<smiles>OB(O)c1ccc([Y](c2ccc(B(O)O)cc2)(c2ccc(B(O)O)cc2)c2ccc(B(O)O)cc2)cc1</smiles>

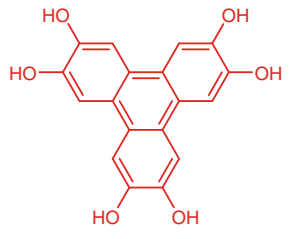

2

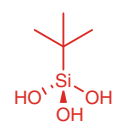<smiles>Oc1cc2c(cc1O)C#Cc1cc(O)c(O)cc1C#Cc1cc(O)c(O)cc1C#C2</smiles>

b

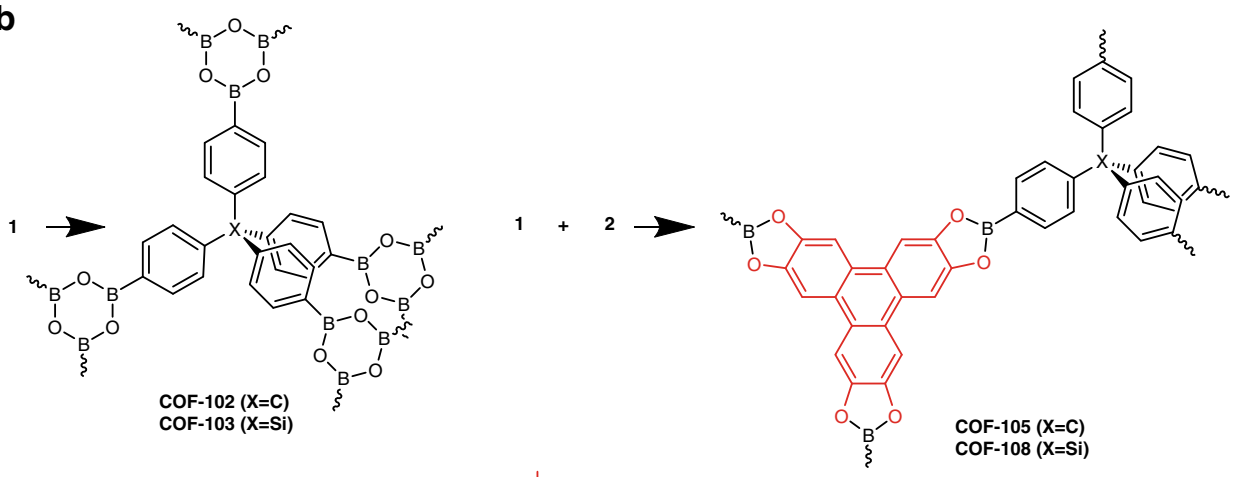<smiles>Cc1ccc(B2O[B-]3(c4ccc(C)cc4)O[Si]4(C(C)(C)C)O[Si](C(C)(C)C)(O2)O[Si](C(C)(C)C)(O3)O4)cc1</smiles>

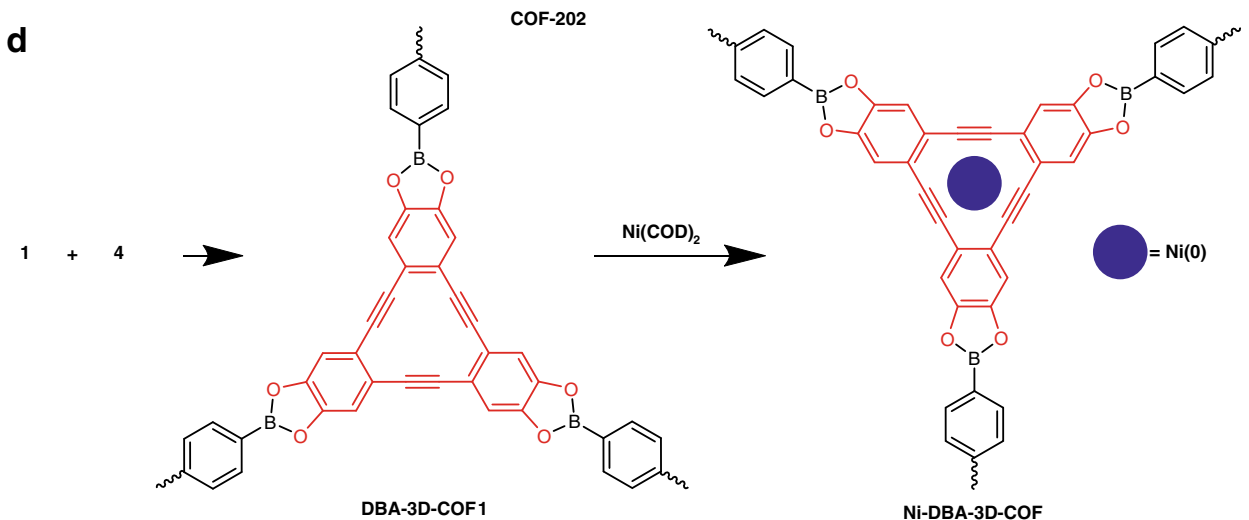

Fig. 2 Precursors and structures of 3D COFs containing B-O linkages. a Building blocks employed for B-O linked 3D COF construction. b Synthetic pathway of COF-102, COF-103, COF-105, and COF-108, the first reported 3D COFs, based on boroxine and boronate ester linkages (adapted with permission from ref. 32. (c) 2007 AAAS). c Synthetic pathway of COF-202, the only 3D COF based on a borosilicate cage linkage (adapted with permission from ref. 46 . (c) 2008 American Chemical Society). d Synthetic approach for DBA-3D-COF, utilizing a planar triangular catechol-functionalized macrocycle and boronate ester linkage to metalate $\mathrm{Ni}$ and obtain Ni-DBA-3D-COF (adapted with permission from ref. ${ }^{24}$. (c) 2016 American Chemical Society)

later utilized for the encapsulation of a common ionic liquid, 1ethyl-3-methylimidazolium bis(trifluoromethylsulfonyl)imide, in its pores $^{57}$.

Since the first report on COF-300, TAPM has been utilized as the tetrahedral amine building block of choice for a range of new imine-linked 3D COF structures. The first instance of a dynamic 3D COF, LZU-301, was synthesized from condensation of TAPM with (3,3'-bipyridine)-6,6'-dicarbaldehyde (Fig. 4d). As the bipyridine analog of COF-320, LZU-301 exhibits symmetry breaking and lattice expansion upon tetrahydrofuran (THF) solvation, which the authors attributed to the conformation change of the $-\mathrm{C}=\mathrm{N}$ - bond, serving as a "molecular pedal" in the crystal structure ${ }^{58}$. Lin et al. ${ }^{59}$ synthesized a pyrene-based 3D
COF by condensing TAPM with 1,3,6,8-tetrakis(4-formylphenyl) pyrene (Fig. 4e), resulting in a novel structure that featured selective adsorption of $\mathrm{CO}_{2}$ over $\mathrm{N}_{2}$ and emitted yellow-green luminescence, marking the first report of a fluorescent 3D COF. Two porphyrin-based $3 \mathrm{D}$ COFs, 3D-Por-COF and 3D-CuPorCOF, were synthesized from the condensation of TAPM with porphyrin-based tetraaldehydes (Fig. 4f), and exhibited photocatalytic activity towards the generation of highly reactive singlet oxygen under visible light irradiation ${ }^{60}$. A 3D chiral COF, CCOF5 , was synthesized by condensation of TAPM with a chiral tetraaldehyde, which subsequently underwent imine oxidation to afford an amide-linked framework, CCOF-6, that retained the crystallinity and permanent porosity of the parent COF (Fig. 4g). 
a

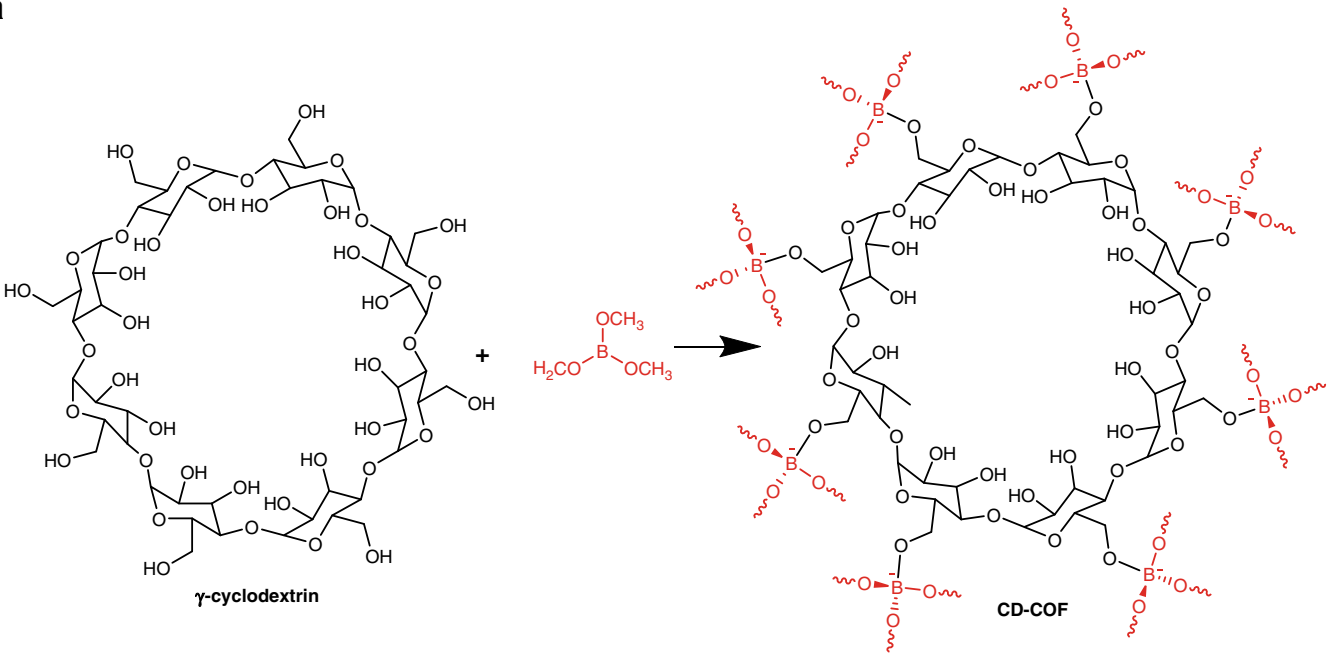

b
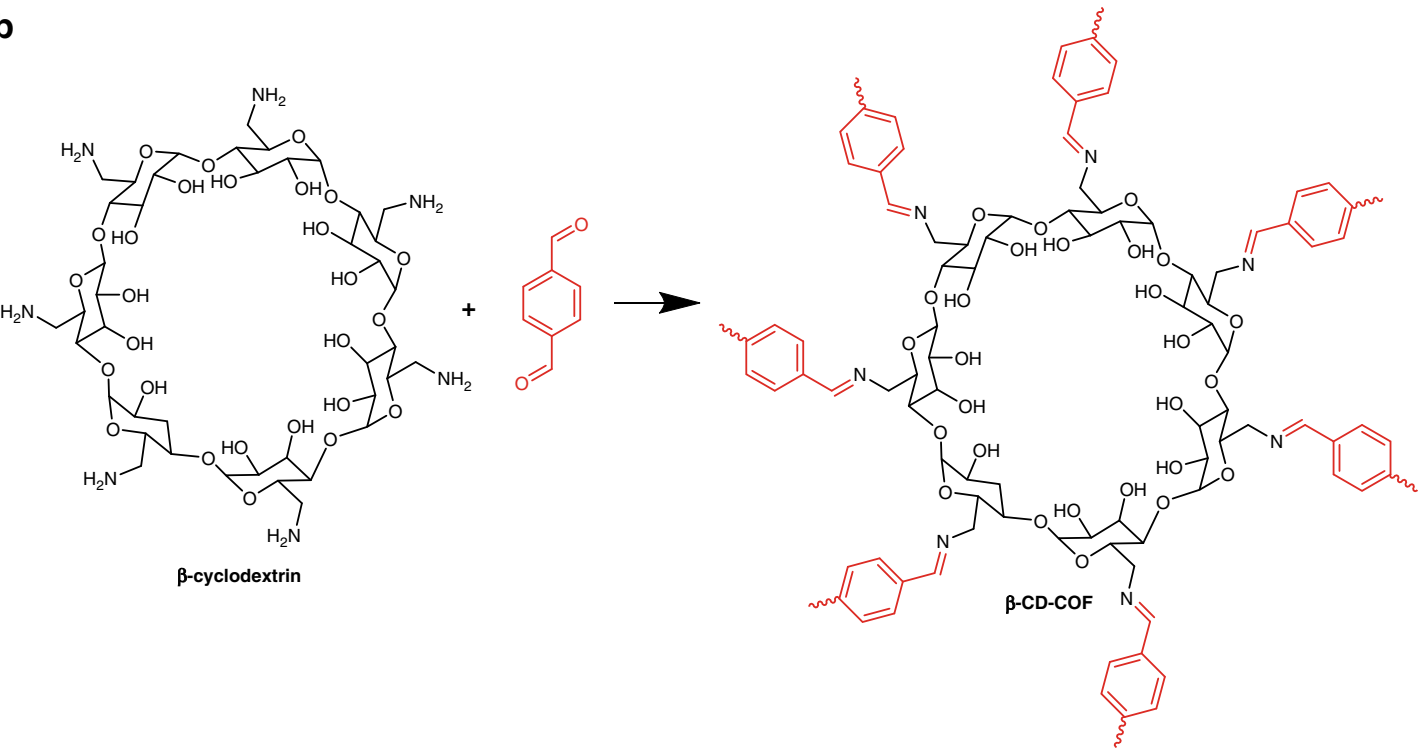

Fig. 3 Synthetic scheme for 3D COFs constructed from flexible aliphatic cyclodextrin. a Synthetic pathway of 3D anionic CD-COF, the first 3D COF constructed from $\gamma$-cyclodextrin utilizing a unique B-O-based spiroborate linkage (adapted with permission from ref. 49 . (C) 2017 John Wiley and Sons). b Synthetic pathway of 3D $\beta$-CD-COF constructed from $\beta$-cyclodextrin and imine linkage (adapted with permission from ref. 66 . (C) 2018 John Wiley and Sons)

Although both CCOF-5 and CCOF-6 could be used as chiral stationary phases (CSP) for high performance liquid chromatography (HPLC), CCOF-6 exhibited superior resolution performance in addition to improved acid and base stability compared to $\mathrm{CCOF}-5^{61}$.

An alternative tetrahedral amine commonly used for 3D COF synthesis is 1,3,5,7-tetraaminoadamantane (TAA). Li et al. ${ }^{62}$ co-condensed TAA with the heterobifunctional 4formylphenylboronic acid and its fluorinated derivative to obtain DL-COF-1 and DL-COF-2, respectively (Fig. 5a), the first 3D COFs to feature dual linkages (imine and boroxine). The bifunctional catalytic capabilities of these two COFs was then demonstrated through acid-base catalyzed one-pot cascade reactions utilizing acidic sites supplied by the boroxine ring and basic sites from the imine bond. While the hydrolytic and oxidative stability of the imine linkage is higher compared to $\mathrm{B}-\mathrm{O}$ linkages found in earlier 3D COFs, they can be further improved by enol-keto tautomerization without affecting the COF structure. This was demonstrated by the Yan group, who reported the first instance of a $3 \mathrm{D} \beta$-ketoenamine COF, BF-
COF-2, in addition to the imine-linked BF-COF-1 (Fig. 5b-c). These COFs were strongly alkaline owing to the high $\mathrm{p} K_{\mathrm{a}}$ of the alkyl amine building blocks (TAA) employed, and the excellent catalytic activity and size selectivity of these COFs for the Knoevenagel condensation reaction was shown ${ }^{63}$. Recently, an alternative adamantane-based tetrahedral amine, 1,3,5,7-tetrakis(4-aminophenyl)-adamantane (TAPA), was employed for $3 \mathrm{D}$ COF synthesis, undergoing reaction with terephthalaldehyde to form COF-DL229 (Fig. 5d). In comparison with COF300 , COF-DL229 possesses higher BET surface area $\left(1762 \mathrm{~m}^{2} / \mathrm{g}\right.$ vs. $1360 \mathrm{~m}^{2} / \mathrm{g}$ ) owing to the more extended radiating arms of TAPA compared with TAPM. Additionally, COF-DL229 achieved an exceptional iodine uptake capacity of $82.4 \mathrm{wt} \%$ in iodine vapor adsorption experiments while simultaneously exhibiting retentiveness and quick release capabilities. The authors also noted that the exclusively organic skeleton of COFs in general are "soft", and observed reduced crystallinity and iodine uptake in recycled COF-DL229, attributed to local structural deformation to fit solid iodine within the pores upon adsorption $^{31}$. 
a<smiles>Nc1ccc(C(c2ccc(N)cc2)(c2ccc(N)cc2)c2cccc(N)c2)cc1</smiles>

5

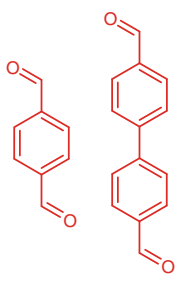

6
7<smiles>O=Cc1ccc(-c2cc(-c3ccc(C=O)cc3)c3ccc4c(-c5ccc(C=O)cc5)cc(-c5ccc(C=O)cc5)c5ccc2c3c54)cc1</smiles>

8

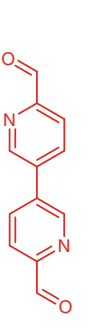

9

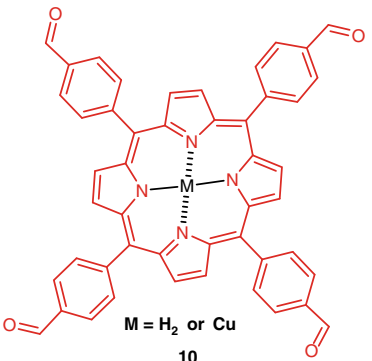

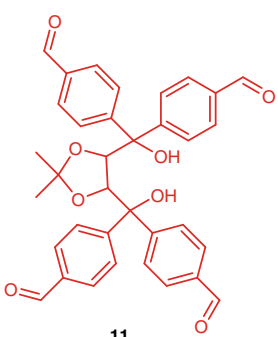<smiles>Cc1ccc(C(c2ccc(C)cc2)(c2ccc(C=Nc3ccc(C(c4ccc(C)cc4)(c4ccc(C)cc4)c4ccc(C)c(C)c4)cc3)cc2)c2ccc(C)c(C)c2)cc1</smiles><smiles>Cc1ccc(C(c2ccc(C)cc2)(c2ccc(C)cc2)c2ccc(N=Cc3ccc(-c4ccc(C(c5ccc(C)cc5)(c5ccc(C)c(C)c5)c5ccc(C)c(C)c5)cc4)cc3)cc2)cc1</smiles>

e<smiles>Cc1ccc(N=Cc2ccc(-c3cc(-c4ccc(/C=N/c5ccc(C)cc5)cc4)c4ccc5c(-c6ccc(/C=N/c7ccc(C)cc7)cc6)cc(-c6ccc(/C=N/c7ccc(C)cc7)cc6)c6ccc3c4c65)cc2)cc1</smiles>

COF-320

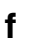<smiles></smiles>

d

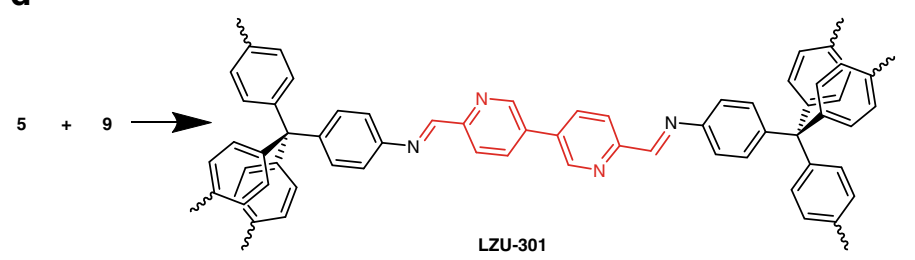
$5+10 \longrightarrow$

g<smiles>Cc1ccc(/N=C/c2ccc(C(O)(c3ccc(C)cc3)C(O)C3CC4(C)OC(C)(C)C4O3)cc2)cc1</smiles>
$5+11 \longrightarrow$<smiles>Cc1ccc(/N=C/c2ccc(C(O)(c3ccc(C)cc3)C(C)OC(C)(C)C)cc2)cc1</smiles>
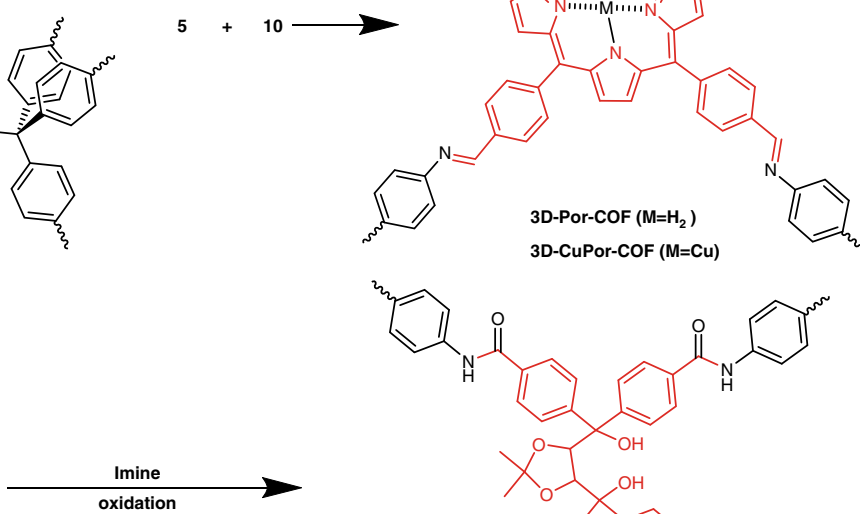<smiles>Cc1ccc(NC(=O)c2ccc(C(O)(c3ccc(C(=O)Nc4ccc(C)cc4)cc3)C(O)(c3ccc(C(=O)Nc4ccc(F)cc4)cc3)C3OC(C)(C)OC3C(O)(c3ccc(C(=O)Nc4ccc(F)cc4)cc3)c3ccc(C(F)(F)F)cc3)cc2)cc1</smiles>

Fig. 4 Building blocks and synthetic schemes for imine-linked 3D COFs constructed from tetrakis-(4-aminophenyl)methane (TAPM, 5). a Aldehyde building blocks employed alongside TAPM for synthesis of imine-linked 3D COFs. Synthetic pathway of b COF-300, the first imine-linked 3D COF (adapted with permission from ref. ${ }^{51}$. (C) 2009 American Chemical Society), c COF-320 (adapted with permission from ref. ${ }^{56}$. (c) 2013 American Chemical Society), d LZU-301, the first 3D COF exhibiting dynamic behavior (adapted with permission from ref. 58. (c) 2017 American Chemical Society), e 3D-Py-COF, the first pyrene-based and first fluorescent 3D COF (adapted with permission from ref. 55. (c) 2016 American Chemical Society), f porphyrin-based 3D-Por-COF and 3D-CuPor-COF (adapted with permission from ref. 60. (c) 2017 American Chemical Society), and $\mathbf{g}$ chiral COFs CCOF-5 and CCOF-6 (adapted with permission from ref. ${ }^{61}$. (c) 2018 American Chemical Society)

Utilizing imine chemistry, Yaghi's group fabricated the first woven COF, COF-505, by employing an aldehyde-functionalized derivative of the copper bis-phenanthroline complex (Fig. 6a). They weaved helical organic threads that complexed to $\mathrm{Cu}(\mathrm{I})$ ions as points of registry, and demonstrated that the structure and topology of COF-505 was retained through demetalation and remetalation, although the demetalated COF-505 did exhibit reduced crystallinity ${ }^{64}$. This work was followed by the synthesis of woven COF-112 from a homogeneous solution, utilizing the gradual in situ deprotection of tert-butyloxycarbonyl-protected amines $(N-B o c)$ to facilitate the formation of nuclei directly from solution and direct growth into crystalline frameworks and avoid precipitation of amorphous insoluble intermediates, while conventional heterogeneous one-pot synthesis resulted in only 
a

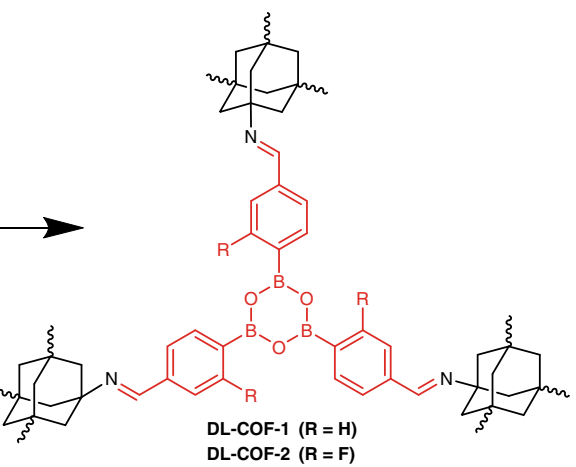

b<smiles>NC1C2(N)CC3(N)CC(N)(C2)CC1(N)C3</smiles>
DL-COF-2 (R = F)<smiles>NC12CC3(N)CC(N)(C1)CC(N)(C2)C3</smiles><smiles>[2H]c1c(C=O)c([2H])c(C=O)c(P)c1C=O</smiles><smiles>CCCCCCCC</smiles><smiles>CC12CC3(C)CC(C)(C1)CC(C)(C2)C3</smiles><smiles>C1CCCCC1</smiles><smiles>CC12CC3CC(C1)C(C)(C3)C2</smiles>

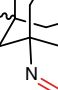
$\mathrm{N}$<smiles>CCc1c(P)c2c(c(P)c1/C=N/C13CC4CC(CC(C)(C4)C1)C3)CCC2</smiles>

BF-COF-1 (R=H)

BF-COF-2 (R=OH)

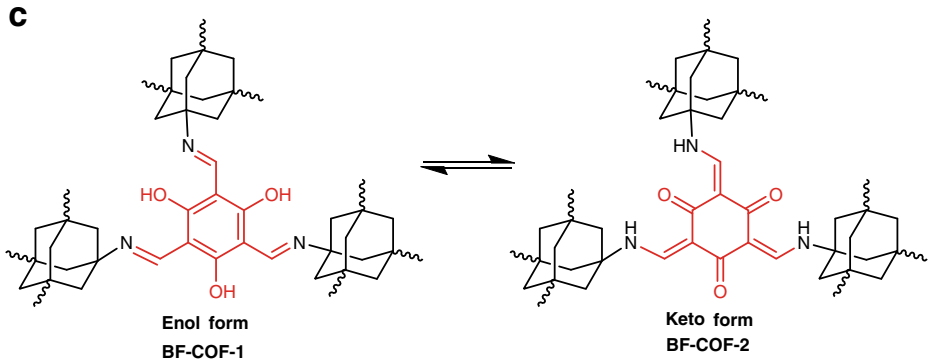

d<smiles>CC12CC3(c4ccc(N)cc4)CC(c4ccc(N)cc4)(C1)CC(c1ccc(N=Cc4ccc(C56CC7(c8ccc(N)cc8)CC(c8ccc(C=O)cc8)(C5)CC(c5ccc(N=Cc8ccc(C=O)cc8)cc5)(C7)C6)cc4)cc1)(C2)C3</smiles>

Fig. 5 Imine-linked 3D COFs synthesized from adamantane-containing tetraamines. a Synthetic approach for DL-COF-1 and DL-COF-2 from 1,3,5,7tetraaminoadamantane (TAA) featuring both imine and boroxine ring linkages (adapted with permission from ref. ${ }^{2}$. (C) 2016 American Chemical Society). b Synthetic pathway of based functionalized BF-COF-1 and BF-COF-2 from TAA. c Enol-keto tautomerization of BF-COF-2, which retains crystallinity and structural integrity (b and c adapted with permission from ref. 63. (c) 2014 John Wiley and Sons). d Synthetic pathway of COF-DL229 from 1,3,5,7-tetrakis (4-aminophenyl)-adamantane (adapted with permission from ref. ${ }^{31}$. (c) 2017 John Wiley and Sons)

amorphous materials (Fig. 6b). Synthesis of a prototype 2D imine COF, LZU-1, utilizing the same protected amine species under rapid microwave heating resulted in nanocrystals with a surface area $\left(729 \mathrm{~m}^{2} / \mathrm{g}\right)$ more than 1.5 times that of the original report $\left(457 \mathrm{~m}^{2} / \mathrm{g}\right)$, and the fabrication of LZU-1 thin films on silicon substrates was also demonstrated through homogeneous synthesis $^{65}$. An imine-linked 3D COF based on $\beta$-cyclodextrin ( $\beta$-CDCOF) was also synthesized (Fig. 3b) ${ }^{66}$.

Recently, Guan et al. ${ }^{67}$ demonstrated the fast ionothermal synthesis of 3D COFs under ambient temperature and pressure, synthesizing three $3 \mathrm{D}$ ionic liquid-containing COFs (3D-ILCOFs) from tetrakis(4-formylphenyl)methane (TFPM) and diamines instead of the more prevalent tetraamine and dialdehyde combination (Fig. 7). By utilizing the ionic liquid 1butyl-3-methylimidazolium bis((trifluoromethyl)sulfonyl)imide as both solvent and catalyst for the imine formation reaction, the authors were able to synthesize crystalline solids at ambient temperature and pressure within $12 \mathrm{~h}$, significantly faster than the 3-7 days required for the conventional solvothermal method. The ionic liquid can be reused without activity loss, and the 3D-ILCOFs were determined to contain $8-12 \%$ ionic liquid in their pores, which contributed to the high adsorption selectivity for $\mathrm{CO}_{2} / \mathrm{N}_{2}$ and $\mathrm{CO}_{2} / \mathrm{CH}_{4}$. This works presents an exciting new stage in 3D COF synthesis, and will be discussed in greater detail in the Perspective section below.

Other linkages. In addition to the $\mathrm{B}-\mathrm{O}$ and imine linkages, a handful of other linkages have also been utilized for constructing $3 \mathrm{D}$ COFs (Fig. 8). Beaudoin et al. ${ }^{68}$ reported COFs constructed using reversible self-addition polymerizations of nitroso groups to 
$\mathbf{a}$

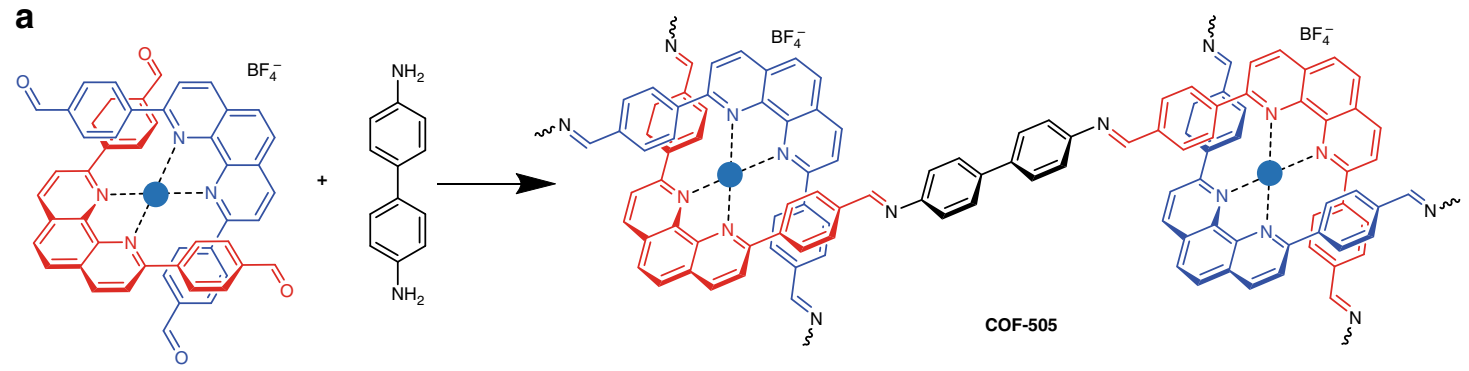

$=\mathrm{Cu}(\mathbf{l})$

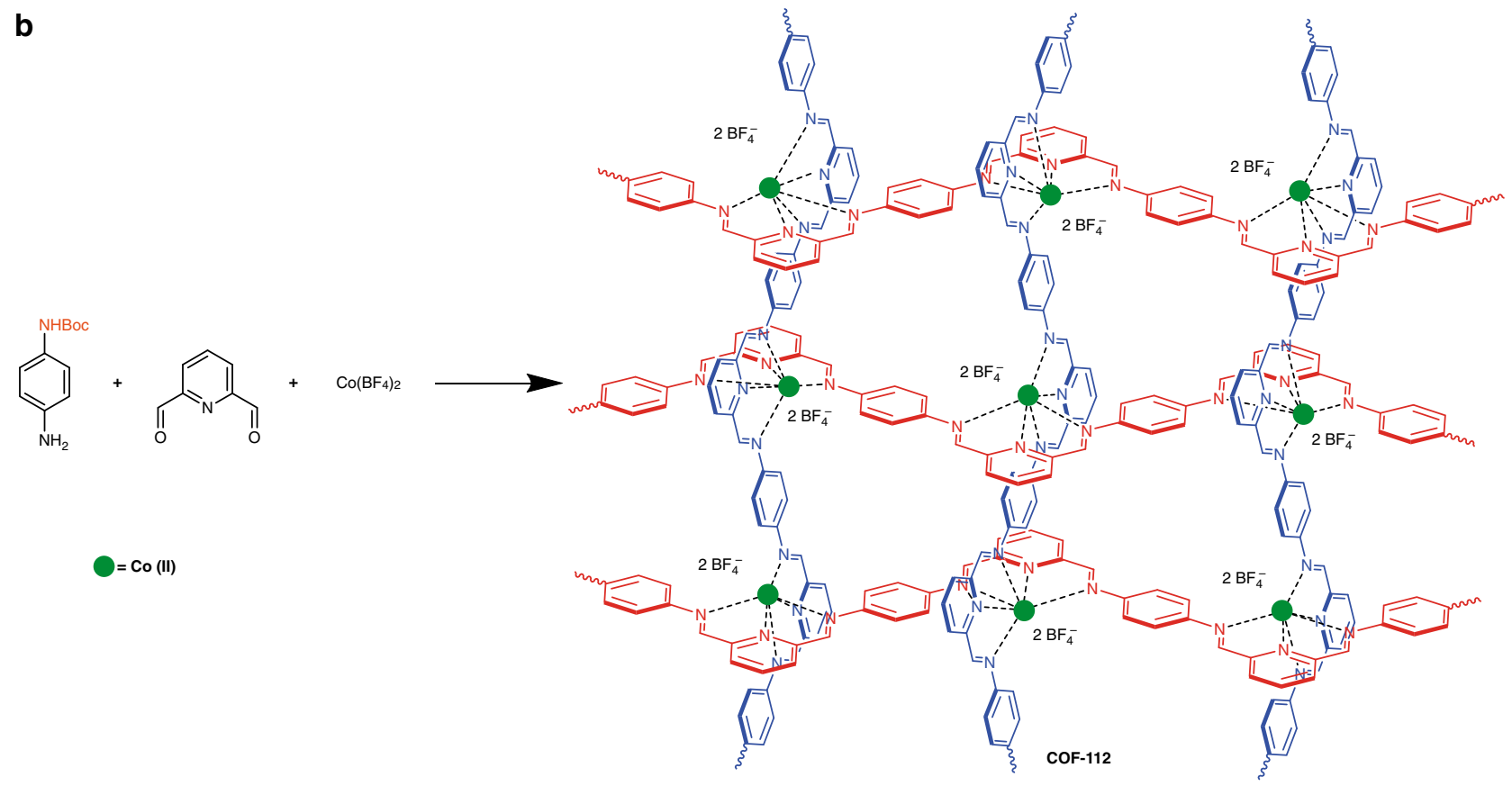

Fig. 6 Synthetic schemes for woven COFs. a Synthetic scheme of woven COF-505 (adapted with permission from ref. 64 . (c) 2016 AAAS). b Synthetic approach of woven COF-112 utilizing in situ deprotection of Boc-protected amine monomer to avoid precipitation of amorphous intermediates and to form crystalline frameworks (adapted with permission from ref. ${ }^{65}$. (c) 2017 American Chemical Society)

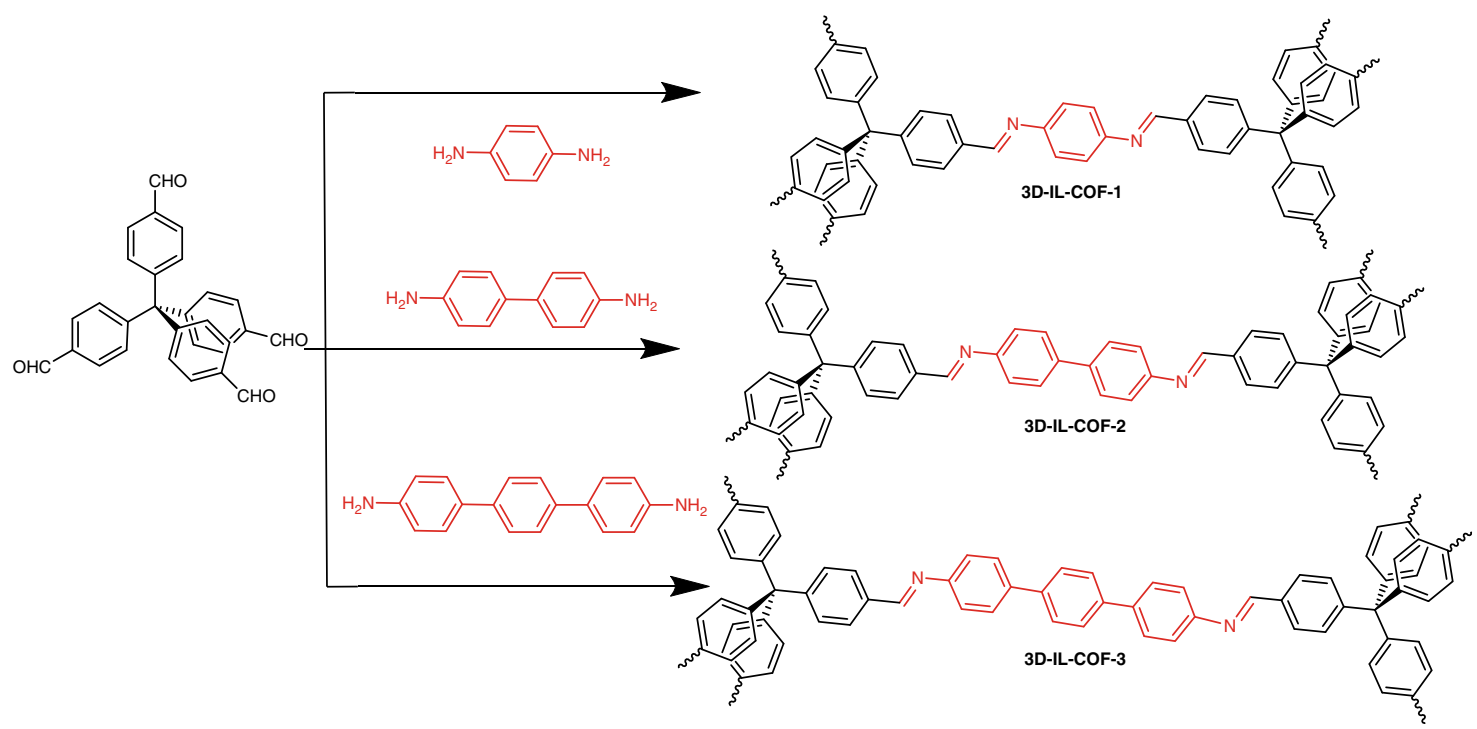

Fig. 7 Synthetic scheme 3D COFs obtained through ionothermal synthesis. 3D-IL-COFs 1-3 were synthesized from tetrakis-(4-formylphenyl)methane and aromatic diamines of varying lengths under ambient temperature and pressure using ionothermal reaction conditions (adapted with permission from ref. 67. (c) 2018 American Chemical Society) 
a

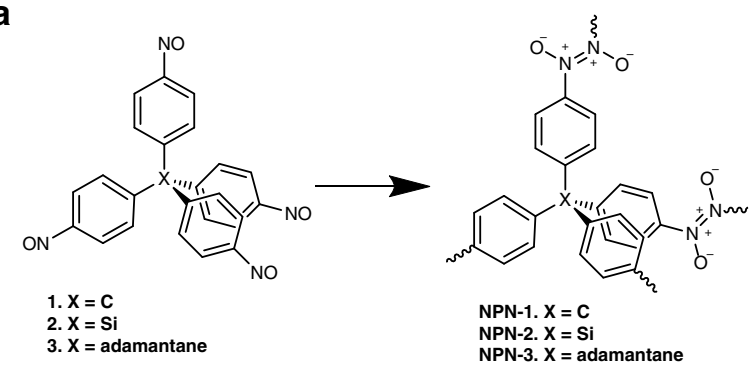

b<smiles>Cc1ccc(-c2nc(-c3ccc(C)cc3)nc(-c3ccc(C(c4ccc(C)cc4)(c4ccc(C)cc4)c4ccc(C)c(C)c4)cc3)n2)cc1</smiles>

C

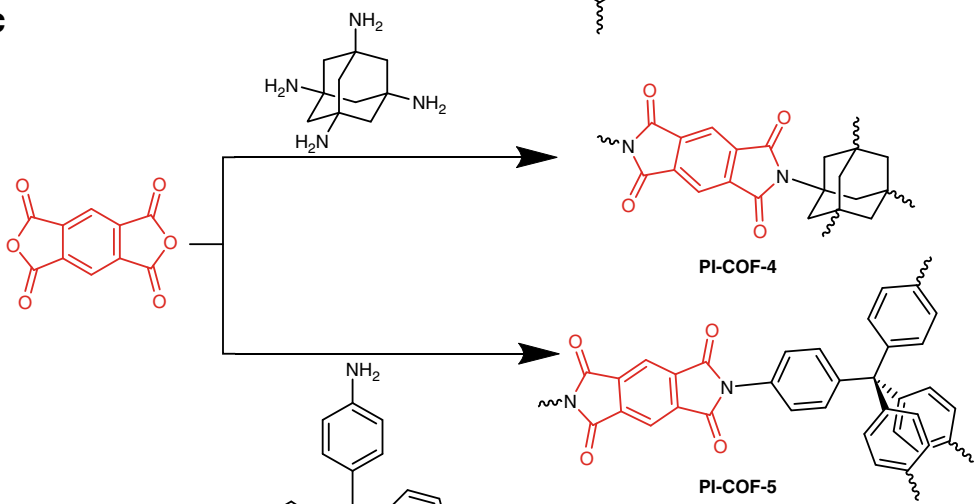

d<smiles>Nc1ccc(CCc2ccc(N)c(N)c2)cc1</smiles><smiles>C1CCCC1</smiles><smiles>Oc1cc2c3cc(O)c(O)cc3c3cc(O)c(O)cc3c2cc1O</smiles><smiles>CO[Si](OC)(OC)OC</smiles>

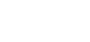


delivery ${ }^{70}$. Yahiaoui et al. ${ }^{71}$ recently reported the first synthesis of a three-coordinated anionic 3D COF, based on novel reversible $\mathrm{Si}-\mathrm{O}$ chemistry and resulting in a topology that had not been previously reported for 3D COFs (Fig. 8d). This work also marked the first instance of a 3D COF that did not require tetrahedral building blocks. Several other linkages, including hydrazones, azines, and imides, have been utilized for the synthesis of $2 \mathrm{D} \mathrm{COFs}$, but have yet to be explored for $3 \mathrm{D} \mathrm{COFs}{ }^{50}$.

Post-synthetic modification of 3D COFs. The organic nature of COFs affords facile chemical tunability, leading to immense structural and functional diversity compared to their inorganic counterparts. As such, modification and functionalization of the COF structure becomes a crucial step towards tailored applications. Excluding the use of different monomers or alternative linkages, post-synthetic modification (PSM) is the most common approach to COF functionalization. A common PSM method is embedding reactive functionalities into constituent monomers and applying subsequent chemistry to those groups upon completion of the initial COF synthesis. Using this approach, Dichtel and coworkers carried out a series of interior functionalization studies on COF-102. Utilizing a monomer-truncation strategy, they co-condensed the tetrahedral boronic acid building block of COF-102 with a truncated version in which one of the four arylboronic acid groups was replaced with a dodecyl or allyl group, obtaining internally functionalized $\mathrm{COF}$ structures with the truncated moieties distributed throughout the material that retains the crystallinity and high surface area of COF-102 (Fig. 9a) 72. The introduced allyl group was subject to post-synthetic functionalization using thiol-ene chemistry, achieving full conversion of the allyl group while retaining crystallinity and permanent porosity (Fig. 9b) ${ }^{73}$.

Dichtel and coworkers ${ }^{74}$ also explored the functionalization of COF-102 utilizing the monofunctional tolylboronic acid instead of the trigonal truncated monomers of the previous studies (Fig. 9c), and found that COF-102-tolyl crystallized even in the presence of up to 36 equivalents of tolylboronic acid relative to the tetra-boronic acid and demonstrated incorporation of up to $36 \mathrm{~mol} \%$ of tolylboronic acid into the framework while maintaining crystallinity and porosity, which the authors postulated as a fundamental limit for the COF-102 structure. Similarly, Lu et. al ${ }^{75}$ demonstrated the synthesis of a carboxylated, imine-linked 3D-COOH-COF by performing PSM on a 3D hydroxy-functionalized COF (Fig. 9d). An alternative PSM technique for imine-linked COFs is the direct modification of the imine bond. For example, Han et al. ${ }^{61}$ converted their iminelinked 3D COF CCOF-5 into an amide-linked CCOF- 6 by oxidation of the imine bonds, achieving enhanced chemical stability compared to its predecessor (Fig. 4g). Reversible enolketo tautomerization has also been shown by the Yan group in the conversion between BF-COF-1 and BF-COF-2 (Fig. $5 \mathrm{c})^{63}$.

A number of other chemistries have been employed for PSM of 2D COFs, including copper(I)-catalyzed azide-alkyne cycloaddition $^{76,77}$, acylation of phenol groups ${ }^{44}$, and the ringopening of succinic anhydride with phenol groups to form carboxylic acids ${ }^{78}$; these chemistries are wholly transferrable to 3D COFs. Some alternative PSM techniques have also been implemented in 2D COFs. For example, Chen et al. ${ }^{79}$ highlighted the ability to lock 2D COF networks with hydrogen bonds by introducing hydroxy groups into the terephthalaldehyde linker, which subsequently form hydrogen bonds with imine-centered nitrogen atoms. Structural modification of COFs has also been achieved through induction of cis-trans isomerization to facilitate a reversible conformation change. Zhang et al. ${ }^{80}$ demonstrated this concept utilizing the trans-cis photoisomerization of azobenzene, using an azobenzene diboronic acid to produce a boronate ester-linked 2D Azo-COF that underwent photoisomerization under $365 \mathrm{~nm}$ UV irradiation. The dynamic behavior of the 3D LZU-301, highlighted in the previous section, can be similarly attributed to an imine bond conformation change ${ }^{58}$. An important consideration for applying PSM to 3D COFs is the reaction site accessibility within the pores. Modification of internal structure of 3D frameworks requires ready access of reactant species to internal functionalization sites, which may impose additional barriers for the translation of $2 \mathrm{D}$ COF PSM techniques to their 3D counterparts. In contrast, PSM techniques developed for 3D MOFs, such as the introduction of polymerizable methacrylamide functionalities into MOF UiO$66^{16}$ or the removal of photolabile $o$-nitrobenzyl pendant groups $^{81}$, could be more readily implemented in 3D COFs.

\section{Challenges in 3D COF development}

Disparity between 2D and 3D frameworks. Although there have been notable advances in the field of 3D COFs, progress persistently lags behind that of 2D COFs. For example, several dynamic covalent linkages that have been employed for synthesizing $2 \mathrm{D}$ COFs, such as hydrazones and azines, have yet to see similar utilization for 3D $\mathrm{COFs}^{50}$. Moreover, compared to the more abundant and systematic studies on the synthesis, modification, and application of $2 \mathrm{D}$ COFs, the current research in $3 \mathrm{D} \mathrm{COFs}$ revolves around the synthesis and characterization of novel structures and preliminary applications that arise from the unique traits of each structure.

Addressing the crystallization problem. A major factor behind this COF development disparity is the so-called "crystallization problem", originating from the inherent conflict between the use of robust covalent bonds to form stable crystalline frameworks, which necessitates the formation of reversible or rearrangeable linkages to facilitate error-correction, and the tendency of covalent bonds to form irreversible interactions ${ }^{82}$. Even in these dynamic covalent bond-forming reactions, the kinetic trapping of amorphous networks often occurs owing to the bulkiness and rigidity of the monomeric precursors impeding proximity between reactive groups, precluding further network rearrangement into the desired crystalline framework. Indeed, in contrast to $2 \mathrm{D}$ COFs where reaction sites are readily accessed and van der Waals interactions from $\pi$-orbital overlap provide additional driving force outside of covalent bonds towards the stacked layer structure, the synthesis of 3D COFs relies solely on inaccessible covalent interactions for the rearrangement of its rigid building blocks ${ }^{61}$. Nevertheless, as the synthesis of contemporary 3D COFs have relied primarily on boronic acid and imine condensation reactions, the formation of stoichiometric quantities of water provides a convenient factor in the mediation of the reaction equilibrium, which can be accomplished by using water immiscible solvents or controlling reactor pressure ${ }^{82}$.

To date, the synthesis of 3D COFs has primarily been performed under heating, commonly at $120^{\circ} \mathrm{C}$ but even at temperatures of up to $160^{\circ} \mathrm{C}^{70}$, either via the predominant solvothermal approach for extended periods or under rapid microwave heating, providing additional energy for covalent bond rearrangement as well as the necessary mobility for the COF building blocks to achieve the desired connectivity. This is essentially a "brute force" method to overcome the crystallization problem, and these reaction conditions impose limitations on the utility of 3D COFs. Reactions that proceed over multiple days may be too slow for industrial relevance, and heating to high temperatures reduces functional group compatibility and precludes certain applications such as the encapsulation of folded biomacromolecules. The development of milder conditions capable of rapidly yielding crystalline materials will be key to unlocking the potential of $3 \mathrm{D}$ COFs for a broader range of applications, and researchers have just begun to explore various 
a<smiles>OB(O)c1ccc(C(c2ccc(B(O)O)cc2)(c2ccc(B(O)O)cc2)c2ccc(B(O)O)cc2)cc1</smiles>

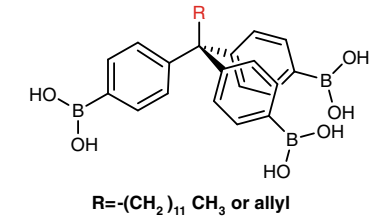

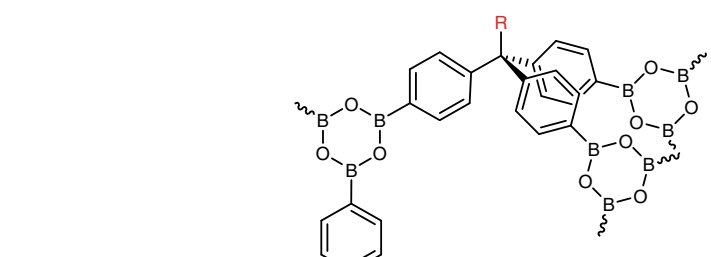

b<smiles>C=CCC(CC)(c1ccc(B2OB(C)OB(C)O2)cc1)c1ccc(B2OB(C)OB(C)OB2OB(C)OB2OB(C)OB(C)O2)cc1</smiles><smiles>CB1OB(C)OB(c2ccc(C(C)C(C)C(C)C)cc2)O1</smiles><smiles>CCCCc1ccccc1BOC</smiles>

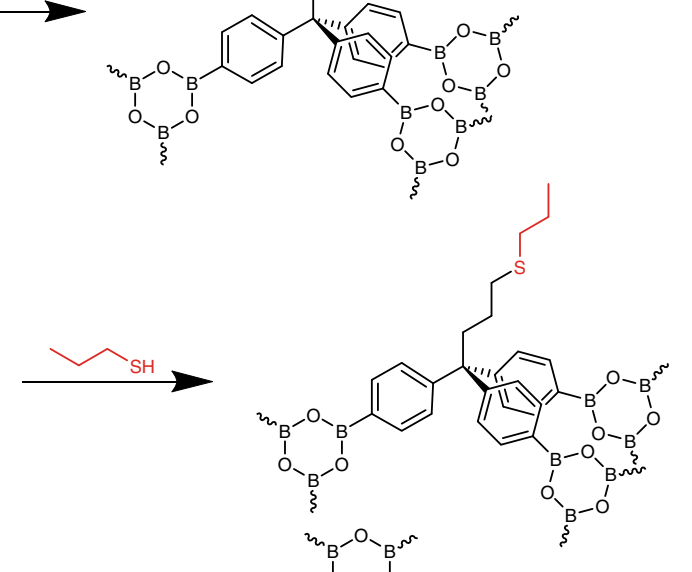

c<smiles>OB(O)c1ccc(C(c2ccc(B(O)O)cc2)(c2ccc(B(O)O)cc2)c2ccc(B(O)O)cc2)cc1</smiles><smiles>Cc1ccc(B(O)O)cc1</smiles>

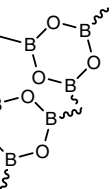<smiles>CB1OB(c2ccc(CC/C=C\COO)cc2)O[C@@H](C(C)C)O1</smiles><smiles>[B]1CCCO1</smiles><smiles>CCCCCCC(C)c1ccc(B(OC)OC)c(B2OB(C)OB(OC)O2)c1</smiles>

d<smiles>C=Cc1ccc(C(c2ccc(C=O)cc2)(c2ccc(C=O)cc2)c2ccc(C=O)c(C=O)c2)cc1</smiles><smiles>Cc1ccc(/C=N/c2ccc(-c3ccc(/N=C/c4ccc(C)cc4)c(OC(=O)CCC(=O)O)c3)cc2OC(=O)CCC(=O)O)cc1</smiles>

Fig. 9 Synthetic schemes for the internal functionalization of model framework COF-102. a Functional group incorporation into COF framework through the use of a truncated version of the tetrahedral boronic acid building block (adapted with permission from ref. 72 . (C) 2012 John Wiley and Sons). b Postsynthetic functionalization of a reactive allyl group, initially introduced by a truncated monomer, via the radical-mediated thiol-ene addition reaction (adapted with permission from ref. 73 . (c) 2013 Royal Society of Chemistry). c Functionalization using monofunctional tolylboronic acid (adapted with permission from ref. ${ }^{74}$. (c) 2014 Elsevier). d Preparation of 3D carboxy-functionalized 3D-COOH-COF through post-synthetic modification of hydroxyfunctionalized 3D-OH-COF (adapted with permission from ref. ${ }^{75}$. (c) 2018 John Wiley and Sons)

means of overcoming the crystallization problem outside extended heating.

In the case of imine-linked COFs, a mechanistic study of the 2D TAPB-PDA COF has revealed that the amine and aldehyde monomers very quickly precipitate into amorphous networks, which then slowly rearrange into crystalline networks after extended heating, demonstrating imine exchange as the rate limiting step in COF formation ${ }^{83}$. An acid catalyst, commonly acetic acid, is often employed to promote imine formation and exchange $^{19}$; however, acetic acid is a relatively inefficient catalyst for transamination ${ }^{84}$. Lewis acidic rare earth metal triflates are promising alternative catalysts owing to their effectiveness in mediating imine exchange reactions, tolerance of both aqueous and organic environments, and functional group compatibility ${ }^{84}$. These metal triflates, particularly scandium triflate $\left(\mathrm{Sc}(\mathrm{OTf})_{3}\right)$, have recently been shown to catalyze the formation of crystalline 2D imine-linked COFs at room temperature in as little as $10 \mathrm{~min}$ while yielding improved material properties such as BET surface area $\left(2175 \mathrm{~m}^{2} / \mathrm{g} \text { vs. }<1000 \mathrm{~m}^{2} / \mathrm{g} \text { from the solvothermal method }\right)^{84}$. Although similar studies have yet to be described for 3D COFs, the utilization of improved catalysts, such as metal triflates, for network rearrangement is a promising approach towards milder 
reaction conditions. Of course, the reduced driving force for $3 \mathrm{D}$ COF network formation suggests that the directly applicability of conditions identified for the 2D TAPB-PDA COF $\left(2 \% \mathrm{Sc}(\mathrm{OTf})_{3}\right.$ loading, $10 \mathrm{~min}$ at room temperature $)^{84}$ is unlikely, and some combination of elevated temperature, extended reaction duration, and higher catalyst loading may be required to induce formation of a crystalline, imine-based 3D network. Interestingly, whereas low rare earth metal triflate concentrations catalyze imine rearrangement reactions, at raised concentrations these compounds no longer act catalytically and instead affect the reaction equilibrium to favor the precursor reactants ${ }^{85}$, an attribute likely to find synthetic utility in mediating framework crystallinity and post-synthetic modification.

Notably, the room temperature synthesis of $3 \mathrm{D}$ COFs has recently been demonstrated. Guan et al. ${ }^{67}$ used an ionic liquid as both solvent and catalyst to obtain highly crystalline, iminelinked 3D COFs in as little as three minutes at ambient temperature and pressure. Although this ionothermal approach holds promise, several aspects of this approach remain subject to optimization. The 3D-IL-COFs synthesized in this study contained $8-12 \%$ ionic liquid in their pores, potentially contributing to their relatively low internal surface areas. For example, 3D-IL-COF-1 exhibited a BET surface area of $517 \mathrm{~m}^{2}$ / $\mathrm{g}^{67}$, whereas COF-300, whose structure differed from 3D-ILCOF-1 only by the directionality of the imine bonds, offered a BET surface area of $1360 \mathrm{~m}^{2} / \mathrm{g}^{51}$, suggesting that this ionothermal synthetic approach may compromise the internal surface area of $3 \mathrm{D}$ COFs, one of their most important characteristics. Moreover, applications of $3 \mathrm{D}$ COFs invariably revolve around host-guest interactions within the pores, and the apparent retention of ILs presents an additional complication in the compatibility of the guest species. Finally, the applicability of this approach to generic imine-based frameworks still needs to be demonstrated. Whereas the three 3D-IL-COFs synthesized in this paper possessed similar structures, differing only by the number of phenyl rings in the linear diamine linker ${ }^{67}$, further investigation is necessary to determine the breadth of ionothermal synthesis utility. In a separate study, $\mathrm{Ma}$ et al. also demonstrated the ability to synthesize single crystalline COF-300 at room temperature by utilizing aniline as a nucleation inhibitor and extending the reaction duration to 30-40 days, and elucidated greater insight into the COF structure through single-crystal analysis ${ }^{53}$. Unfortunately, the extended reaction times required by this strategy would likely curtail adoption of the resultant 3D COFs for industrial applications.

The lack of control over the rapid precipitation of amorphous intermediates during COF synthesis can lead to kinetic trapping of said intermediates, preventing the rearrangement necessary to form a crystalline structure. Limiting the concentration of available monomers in the reaction through controlled addition of reactants or through in situ deprotection or generation of reactant species can contribute to reaction rate control, mediating the rate of crystal nucleation and growth, and ultimately overcoming kinetic trapping. The Dichtel group reported the successful growth of the first discrete colloidal 2D boronate esterlinked COF-5 particles via gradual monomer addition, whereas faster addition led predominantly to the formation of new particles with smaller crystalline domains ${ }^{86}$. Similarly, Yuan et al. ${ }^{87}$ demonstrated the use of heterogeneous $\mathrm{SiO}_{2}$ seeds coated with a thin layer of amorphous Schiff-base polymer from which the imine-linked 2D COF-LZU-1 network was grown, suppressing the rapid precipitation of amorphous materials and promoting crystallization. The frameworks were obtained in significantly shorter time frame than conventional solvothermal methods and exhibited high crystallinity and surface area after the $\mathrm{SiO}_{2}$ particles were etched away. Additionally, interfacial crystallization, multiple demonstrations of which have been reported for $2 \mathrm{D} \mathrm{COFs}^{88,89}$, may be applicable to $3 \mathrm{D}$ COFs. For example, Li et al..$^{90}$ demonstrated a novel approach to yield highquality $2 \mathrm{D} \mathrm{COF}$ nanosheets at the interface of two miscible organic solvents, each of which contained one of the two constituent monomers in solution, by adding a low density solvent interlayer as a buffer between the two main solvent layers. Alternatively, in situ deprotection can also control monomer release rate and precipitation, an approach that even enables the use of precursor monomers bearing multiple, covalently coreactive functional group types. In situ deprotection of catechol was previously reported for the incorporation of insoluble and unstable building blocks in boronate ester COF synthesis ${ }^{91}$, and in situ deprotection of certain reactant functional groups can curtail the condensation reaction rate and hence mediate the rate of precipitation to avoid kinetic trapping ${ }^{92}$. Yaghi's group recently applied this concept to woven $3 \mathrm{D}$ COFs, employing in situ deprotection of Boc-protected amine monomers to slow down the initial imine condensation and synthesize the woven COF-112 homogeneously without the precipitation of amorphous intermediates, using trifluoroacetic acid (TFA) as a dual-role catalyst. This approach also afforded greater control over COF morphology, allowing the synthesis of uniform nanocrystals and thin films based on 2D imine-linked COF LZU-1 ${ }^{65}$. Similarly, Yahiaoui et al. ${ }^{71}$ utilized the in situ generation of silicon source tetramethoxysilane (TMOS) by gradual disproportionation of methyltrimethoxysilane to synthesize SiCOF-5, while direct condensation of constituent monomers HHTP and TMOS did not yield a crystalline framework. Establishing more precise control over the monomer availability in the reaction system, either through slow addition, heterogeneous nucleation, or in situ deprotection or generation of constituent species, will likely prove indispensable to circumvent kinetic trapping for a broad range of 3D COF syntheses.

Deficient systematic understanding. Another notable issue that has plagued the COF community is the fragmentary understanding of both their inherent structure and the contributions of COF synthetic conditions, particularly for 3D COFs. To date, most 3D COF synthetic efforts have yielded amorphous or polycrystalline products, and their crystalline morphologies have been determined by PXRD; however, elucidation of the precise structure, including atomic positions, interpenetration, and pore guest arrangement, has proven challenging. This dilemma may be resolved by the construction of single crystals of 3D COFs, enabling a more definitive understanding of their structure via SCXRD $^{53}$. Beaudoin et al. ${ }^{68}$ synthesized monocrystalline COFs by utilizing reversible, self-addition polymerization of tetrahedral nitroso compounds to afford diamondoid azodioxy networks which were then subject to SCXRD characterization, enabling elucidation of the location and bonding of essentially every atom. $\mathrm{Ma}$ et al. ${ }^{53}$ further demonstrated the characterization utility of single crystalline COFs by employing single crystals of COF-300 to resolve prior ambiguity regarding their degree of interpenetration as well as structural distortion upon hydration. These reports represent an important direction in the field of 3D COFs, and continued emphasis on single crystalline 3D COF synthesis will enable definitive resolution of their structures.

The influence of 3D COF synthetic conditions remains poorly understood. Duncan et al. ${ }^{19}$ conducted a study on the kinetic and thermodynamic factors in imine-linked 3D COF synthesis, and found that acetic acid is a crucial component of the reaction, acting as both a catalyst and a co-solvent. In addition, they evaluated the suitability of a range of organic solvents for synthesis and reported that dimethylformamide and THF were the most suitable solvents for obtaining highly cross-linked 
materials, although the polymer networks they obtained remained amorphous $^{19}$. Dichtel's mechanistic study of the crystallization process of $2 \mathrm{D}$ imine-linked COFs provided the first real insight into the synthetic process, and successfully elucidated the mechanism of rapid amorphous precipitation followed by gradual rearrangement into crystalline structures. In addition, the crucial role of acetic acid and water in inducing crystallinity and maintaining high yields was shown ${ }^{83}$. Li et al. ${ }^{93}$ used a kinetic Monte Carlo model to describe the nucleation and growth of $2 \mathrm{D}$ COF-5 from solution which agrees with experimentally measured growth kinetics and showed that nucleation and growth processes could be described by the same model, which could lead to informed 2D COF syntheses and improved control over nucleation and growth. Similar insight into 3D COF synthesis would offer improved understanding and control of the crystallization process.

Other than the aforementioned reaction conditions, additional factors affecting the synthesis of 3D COFs need further elucidation. For example, whereas COF-300 is typically synthesized from a suspension of starting materials, homogeneous starting conditions were reported to yield the best results in a boronate ester 3D COF synthesis ${ }^{24,51}$. A controlled variable study comparing 3D COF synthesis from a suspension or homogeneous solution would inform the influence of these initial conditions. Additionally, COF-300 synthesis has required freeze-pump-thaw degassing and subsequent reaction vessel flame sealing prior to heating ${ }^{51}$, where raised pressure through sealing the reaction vessel has been touted as key to obtaining a crystalline solid ${ }^{82}$. This methodology has been universally adopted for 3D COFs synthesized by the solvothermal method; however, overpressure has proven unnecessary for the synthesis of COFs containing $\mathrm{B}-\mathrm{O}$ linkages, as an open reaction vessel with a reflux condenser attached for collecting water achieved similar results as a sealed reaction vessel in microwave synthesis ${ }^{45}$. Since water plays a similar role in mediating the reaction equilibrium in both boronic acid condensation and imine condensation, one might conclude that a sealed reaction vessel serves as a convenient method for retaining water in the system, but it is not the only way of doing so. Even if the use of a sealed reaction vessel is desired, degassing is potentially unnecessary. Again, controlled variable experiments comparing 3D COF synthesis from a degassed and pressurized versus simply a sealed reaction system, are required to assess this hypothesis, and if confirmed these findings can contribute to simplification of the established 3D COF synthetic process.

Limited topologies and building blocks. A second reason behind the disparity of studies on $2 \mathrm{D}$ and $3 \mathrm{D}$ COFs is the limited variety of available molecular building blocks and consequent network topologies ${ }^{59,71}$. Compared to 2D COFs, which can be constructed from a variety of ditopic and tritopic linkers, 3D COFs have, with the recent exception of the three-coordinated SiCOF-5 with srs topology, utilized tetrahedral building blocks and adopted a topology belonging to one of five nets (dia, bor, ctn, rra, or pts) $49,59,71$. The chemical diversity of these building blocks is similarly limited, as many 3D COFs based on B-O linkages have been synthesized from tetra(4-dihydroxyborylphenyl)methane, and the majority of imine-linked 3D COFs employed TAPM and TAA as precursors $^{82}$.

From a geometry perspective, tetrahedral nodes are the most direct method of sustaining an infinite 3D cross-linked network. Though employing a non-planar tritopic linker would also result in a non-planar structure, growth in the z-direction may be finite and would not necessarily assemble into a true $3 \mathrm{D}$ framework. Employing higher functionality linkers would conceivably yield 3D frameworks with higher degrees of cross-linking; however, limited functional group accessibility, steric hindrance, and kinetic trapping, which, as discussed earlier, are already present when utilizing tetratopic linkers, would become increasingly prevalent and further impede the synthetic accessibility of crystalline structures. As such, tetratopic linkers are ubiquitously employed as the most straightforward way to sustain an infinite 3D cross-linked network around a tetrahedral node, while avoiding increased steric hindrance and kinetic trapping from monomers with higher order functionalities. This limit in topology and building block diversity has limited the number of attainable 3D COF structures, and novel building block structures must be discovered in addition to employing different linkages and COF structure modifications to access increased compositional and structural complexity.

Controlling network interpenetration. Interpenetration, where the empty space described by the crystalline framework accommodates one or more additional networks ${ }^{94}$ and which can adversely affect pore volume and impact internal surface area ${ }^{95}$, is another aspect of 3D COFs that requires improved understanding and synthetic control. Whereas interpenetration in 3D COFs has typically been determined by structural modeling and unit-cell indexing and is reported as an inherent characteristic, efforts to better understand, characterize, and even modify degrees of interpenetration have been recently reported. For example, the synthesis of an interpenetration isomer was reported for COF300 , representing a preliminary attempt towards modifying the extent of interpenetration in a $3 \mathrm{D} \mathrm{COF}^{52}$. More sophisticated approaches to control COF interpenetration might be derived from techniques successfully implemented in MOF syntheses, such as temperature and monomer concentration control ${ }^{96}$, organic bond structure alteration ${ }^{95}$, rational design of organic building blocks ${ }^{97}$, and incorporation of space-filling, photolabile protecting groups that could be post-synthetically cleaved ${ }^{81}$. These techniques inform the potential for reducing or preventing interpenetration in $3 \mathrm{D}$ COFs and could conceivably be translated to 3D COF synthetic approaches to fully realize their potential as crystalline, controllably porous materials.

\section{Outlook}

Covalent organic frameworks are an extremely promising class of nanoporous material, and 3D COFs, possessing notably higher internal surface area than their 2D counterparts, hold significant appeal for applications including gas storage, gas separation, and catalysis. Unfortunately, 3D COFs suffer from poor synthetic accessibility owing to kinetic trapping, a deficiency in systematic understanding of their syntheses, and limited topologies and building blocks. Kinetic trapping of amorphous networks, or the crystallization problem, presents a significant obstacle in obtaining crystalline 3D frameworks. Nevertheless, the development of more effective catalysts for connectivity rearrangement and enhanced control over the rate of precipitation via nucleation inhibition or gradual introduction of monomer species via controlled addition, in situ deprotection, or interfacial polymerization, hold promise for overcoming the crystallization problem. Improved systematic understanding of the COF synthesis reaction would allow for more precise control over the structures and pursuing further synthesis and characterization of single crystals of a wider variety of COFs and systematic investigation of various reaction parameters should be a primary focus. Moreover, diversifying the limited topologies and building blocks and establishing control over network interpenetration would enable the realization of the full potential of 3D COFs as controllable and easily tunable porous materials. We anticipate that these insights can provide guidance and inform future research efforts in advancing the 3D COF field. 
Received: 23 April 2018 Accepted: 26 November 2018

Published online: 18 December 2018

\section{References}

1. Huang, N., Wang, P. \& Jiang, D. Covalent organic frameworks: a materials platform for structural and functional designs. Nat. Rev. Mater. 1, 16068 (2016).

2. Dogru, M. \& Bein, T. On the road towards electroactive covalent organic frameworks. Chem. Commun. 50, 5531-5546 (2014).

3. Feng, X., Ding, X. \& Jiang, D. Covalent organic frameworks. Chem. Soc. Rev. 41, 6010-6022 (2012).

4. Zhu, L. \& Zhang, Y. -B. Crystallization of covalent organic frameworks for gas storage applications. Molecules 22, 1149 (2017).

5. McKeown, N. B. \& Budd, P. M. Polymers of intrinsic microporosity (PIMs): organic materials for membrane separations, heterogeneous catalysis and hydrogen storage. Chem. Soc. Rev. 35, 675-683 (2006).

6. McKeown, N. B. Polymers of intrinsic microporosity. Int. Sch. Res. Not. 2012 e513986 (2012)

7. Yuan, D., Lu, W., Zhao, D. \& Zhou, H. -C. Highly stable porous polymer networks with exceptionally high gas-uptake capacities. Adv. Mater. 23, 3723-3725 (2011).

8. Jiang, J. -X. et al. Conjugated microporous poly(aryleneethynylene) networks. Angew. Chem. Int. Ed. 46, 8574-8578 (2007)

9. Cooper, A. I. Conjugated microporous polymers. Adv. Mater. 21, 1291-1295 (2009).

10. Jiang, J. -X. et al. Synthetic control of the pore dimension and surface area in conjugated microporous polymer and copolymer networks. J. Am. Chem. Soc. 130, 7710-7720 (2008)

11. Ding, S. -Y. \& Wang, W. Covalent organic frameworks (COFs): from design to applications. Chem. Soc. Rev. 42, 548-568 (2013).

12. Yao, S., Yang, X., Yu, M., Zhang, Y. \& Jiang, J. -X. High surface area hypercrosslinked microporous organic polymer networks based on tetraphenylethylene for CO2 capture. J. Mater. Chem. A 2, 8054-8059 (2014).

13. Zhang, Y. \& Riduan, S. N. Functional porous organic polymers for heterogeneous catalysis. Chem. Soc. Rev. 41, 2083-2094 (2012).

14. Bisbey, R. P. \& Dichtel, W. R. Covalent organic frameworks as a platform for multidimensional polymerization. ACS Cent. Sci. 3, 533-543 (2017).

15. Li, J. -R., Sculley, J. \& Zhou, H. -C. Metal-organic frameworks for separations. Chem. Rev. 112, 869-932 (2012).

16. Zhang, Y. et al. Photoinduced postsynthetic polymerization of a metal-organic framework toward a flexible stand-alone membrane. Angew. Chem. 127, 4333-4337 (2015).

17. Zhou, H. -C., Long, J. R. \& Yaghi, O. M. Introduction to metal-organic frameworks. Chem. Rev. 112, 673-674 (2012).

18. Zhu, Y., Long, H. \& Zhang, W. Imine-linked porous polymer frameworks with high small gas $\left(\mathrm{H}_{2}, \mathrm{CO}_{2}, \mathrm{CH}_{4}, \mathrm{C}_{2} \mathrm{H}_{2}\right)$ uptake and $\mathrm{CO}_{2} / \mathrm{N}_{2}$ selectivity. Chem. Mater. 25, 1630-1635 (2013).

19. Duncan, N. C., Hay, B. P., Hagaman, E. W. \& Custelcean, R. Thermodynamic, kinetic, and structural factors in the synthesis of imine-linked dynamic covalent frameworks. Tetrahedron 68, 53-64 (2012).

20. Czaja, A. U., Trukhan, N. \& Müller, U. Industrial applications of metal-organic frameworks. Chem. Soc. Rev. 38, 1284-1293 (2009).

21. Ben, T. et al. Targeted Synthesis of a porous aromatic framework with high stability and exceptionally high surface area. Angew. Chem. Int. Ed. 48 , 9457-9460 (2009).

22. Côté, A. P. et al. Porous, crystalline, covalent organic frameworks. Science 310, 1166-1170 (2005). First reported synthesis of covalent organic frameworks.

23. Zhao, W., Xia, L. \& Liu, X. Covalent organic frameworks (COFs): perspectives of industrialization. CrystEngComm 20, 1613-1634 (2018).

24. Baldwin, L. A., Crowe, J. W., Pyles, D. A. \& McGrier, P. L. Metalation of a mesoporous three-dimensional covalent organic framework. J. Am. Chem. Soc. 138, 15134-15137 (2016).

25. Mendoza-Cortés, J. L., Han, S. S., Furukawa, H., Yaghi, O. M. \& Goddard, W. A. Adsorption mechanism and uptake of methane in covalent organic frameworks: theory and experiment. J. Phys. Chem. A 114, 10824-10833 (2010).

26. Han, S. S., Furukawa, H., Yaghi, O. M. \& Goddard, W. A. Covalent organic frameworks as exceptional hydrogen storage materials. J. Am. Chem. Soc. 130, 11580-11581 (2008).

27. Klontzas, E., Tylianakis, E. \& Froudakis, G. E. Hydrogen storage in 3D covalent organic frameworks. a multiscale theoretical investigation. J. Phys. Chem. C. 112, 9095-9098 (2008).

28. Cao, D., Lan, J., Wang, W. \& Smit, B. Lithium-doped 3D covalent organic frameworks: high-capacity hydrogen storage materials. Angew. Chem. Int. Ed. 48, 4730-4733 (2009).
29. Tylianakis, E., Klontzas, E. \& Froudakis, G. E. Multi-scale theoretical investigation of hydrogen storage in covalent organic frameworks. Nanoscale 3, 856-869 (2011)

30. Furukawa, H. \& Yaghi, O. M. Storage of hydrogen, methane, and carbon dioxide in highly porous covalent organic frameworks for clean energy applications. J. Am. Chem. Soc. 131, 8875-8883 (2009).

31. Wang, C. et al. A 3D covalent organic framework with exceptionally high iodine capture capability. Chem. Eur. J. 24, 585-589 (2017).

32. El-Kaderi, H. M. et al. Designed synthesis of $3 \mathrm{D}$ covalent organic frameworks Science 316, 268-272 (2007). First report of 3D COFs.

33. Huang, N., Ding, X., Kim, J., Ihee, H. \& Jiang, D. A photoresponsive smart covalent organic framework. Angew. Chem. Int. Ed. 54, 8704-8707 (2015).

34. Alahakoon, S. B. et al. An azine-linked hexaphenylbenzene based covalent organic framework. Chem. Commun. 52, 2843-2845 (2016).

35. Ritchie, L. K., Trewin, A., Reguera-Galan, A., Hasell, T. \& Cooper, A. I. Synthesis of COF-5 using microwave irradiation and conventional solvothermal routes. Microporous Mesoporous Mater. 132, 132-136 (2010).

36. Singh, V., Jang, S., Vishwakarma, N. K. \& Kim, D.-P. Intensified synthesis and post-synthetic modification of covalent organic frameworks using a continuous flow of microdroplets technique. NPG Asia Mater. 10, e456 (2018).

37. Bisbey, R. P., DeBlase, C. R., Smith, B. J. \& Dichtel, W. R. Two-dimensional covalent organic framework thin films grown in flow. J. Am. Chem. Soc. 138, 11433-11436 (2016)

38. Peng, Y. et al. Room temperature batch and continuous flow synthesis of water-stable covalent organic frameworks (COFs). Chem. Mater. 28, 5095-5101 (2016)

39. Jiang, Y. et al. Green, scalable and morphology controlled synthesis of nanofibrous covalent organic frameworks and their nanohybrids through a vapor-assisted solid-state approach. J. Mater. Chem. A 2, 8201-8204 (2014).

40. Medina, D. D. et al. Room temperature synthesis of covalent-organic framework films through vapor-assisted conversion. J. Am. Chem. Soc. 137, 1016-1019 (2015)

41. Ding, S.-Y. et al. Construction of covalent organic framework for catalysis: $\mathrm{Pd} /$ COF-LZU1 in Suzuki-Miyaura coupling reaction. J. Am. Chem. Soc. 133, 19816-19822 (2011)

42. Pachfule, P., Kandambeth, S., Díaz, D. D. \& Banerjee, R. Highly stable covalent organic framework-Au nanoparticles hybrids for enhanced activity for nitrophenol reduction. Chem. Commun. 50, 3169-3172 (2014).

43. Valentino, L., Matsumoto, M., Dichtel, W. R. \& Mariñas, B. J. Development and performance characterization of a polyimine covalent organic framework thin-film composite nanofiltration membrane. Environ. Sci. Technol. 51, 14352-14359 (2017).

44. Zhao, S. et al. Channel-wall functionalization in covalent organic frameworks for the enhancement of $\mathrm{CO}_{2}$ uptake and $\mathrm{CO}_{2} / \mathrm{N}_{2}$ selectivity. RSC $A d v .6$, 38774-38781 (2016)

45. Campbell, N. L., Clowes, R., Ritchie, L. K. \& Cooper, A. I. Rapid microwave synthesis and purification of porous covalent organic frameworks. Chem. Mater. 21, 204-206 (2009).

46. Hunt, J. R., Doonan, C. J., LeVangie, J. D., Côté, A. P. \& Yaghi, O. M. Reticular synthesis of covalent organic borosilicate frameworks. J. Am. Chem. Soc. 130, $11872-11873$ (2008)

47. Liu, X.-Y. et al. Effect of metal catalyst on the mechanism of hydrogen spillover in three-dimensional covalent-organic frameworks. Chin. Phys. B 26 027302 (2017)

48. Kalidindi, S. B., Yusenko, K. \& Fischer, R. A. Metallocenes@COF-102: organometallic host-guest chemistry of porous crystalline organic frameworks. Chem. Commun. 47, 8506-8508 (2011).

49. Zhang, Y. et al. Three-dimensional anionic cyclodextrin-based covalent organic frameworks. Angew. Chem. Int. Ed. 56, 16313-16317 (2017).

50. DeBlase, C. R. \& Dichtel, W. R. Moving beyond boron: the emergence of new linkage chemistries in covalent organic frameworks. Macromolecules 49, 5297-5305 (2016)

51. Uribe-Romo, F. J. et al. A crystalline imine-linked 3-D porous covalent organic framework. J. Am. Chem. Soc. 131, 4570-4571 (2009). Report describing COF-300, the archetypal, imine-linked $3 D$ COF.

52. Ma, T. et al. Observation of interpenetration isomerism in covalent organic frameworks. J. Am. Chem. Soc. 140, 6763-6766 (2018).

53. Ma, T. et al. Single-crystal $\mathrm{x}$-ray diffraction structures of covalent organic frameworks. Science 361, 48-52 (2018). Single crystal $x$-ray study of COF300 , providing deeper structural insight than previously attained.

54. Das, S. \& Ben, T. A [COF-300]-[UiO-66] composite membrane with remarkably high permeability and $\mathrm{H} 2 / \mathrm{CO} 2$ separation selectivity. Dalton. Trans. 47, 7206-7212 (2018).

55. Qian, H.-L., Yang, C. \& Yan, X.-P. Layer-by-layer preparation of 3D covalent organic framework/silica composites for chromatographic separation of position isomers. Chem. Commun. 54, 11765-11768 (2018). 
56. Zhang, Y.-B. et al. Single-crystal structure of a covalent organic framework. J. Am. Chem. Soc. 135, 16336-16339 (2013).

57. Xin, Y., Wang, C., Wang, Y., Sun, J. \& Gao, Y. Encapsulation of an ionic liquid into the nanopores of a 3D covalent organic framework. RSC Adv. 7, 1697-1700 (2017).

58. Ma, Y.-X. et al. A dynamic three-dimensional covalent organic framework. J. Am. Chem. Soc. 139, 4995-4998 (2017).

59. Lin, G., Ding, H., Yuan, D., Wang, B. \& Wang, C. A pyrene-based, fluorescent three-dimensional covalent organic framework. J. Am. Chem. Soc. 138, 3302-3305 (2016).

60. Lin, G. et al. 3D porphyrin-based covalent organic frameworks. J. Am. Chem. Soc. 139, 8705-8709 (2017).

61. Han, X., Huang, J., Yuan, C., Liu, Y. \& Cui, Y. Chiral 3D covalent organic frameworks for high performance liquid chromatographic enantioseparation. J. Am. Chem. Soc. 140, 892-895 (2018).

62. Li, H. et al. Three-dimensional covalent organic frameworks with dual linkages for bifunctional cascade catalysis. J. Am. Chem. Soc. 138, 14783-14788 (2016).

63. Fang, Q. et al. 3D microporous base-functionalized covalent organic frameworks for size-selective catalysis. Angew. Chem. Int. Ed. 53, 2878-2882 (2014).

64. Liu, Y. et al. Weaving of organic threads into a crystalline covalent organic framework. Science 351, 365-369 (2016).

65. Zhao, Y. et al. A synthetic route for crystals of woven structures, uniform nanocrystals, and thin films of imine covalent organic frameworks. J. Am. Chem. Soc. 139, 13166-13172 (2017).

66. Wang, R. -Q., Wei, X. -B. \& Feng, Y. -Q. $\beta$-Cyclodextrin covalent organic framework for selective molecular adsorption. Chem. Eur. J. 24, 10979-10983 (2018).

67. Guan, X. et al. Fast, ambient temperature and pressure ionothermal synthesis of three-dimensional covalent organic frameworks. J. Am. Chem. Soc. 140, 4494-4498 (2018). Demonstration of 3D COF synthesis at room temperature.

68. Beaudoin, D., Maris, T. \& Wuest, J. D. Constructing monocrystalline covalent organic networks by polymerization. Nat. Chem. 5, 830-834 (2013).

69. Ren, S. et al. Porous, fluorescent, covalent triazine-based frameworks via room-temperature and microwave-assisted synthesis. Adv. Mater. 24, 2357-2361 (2012).

70. Fang, Q. et al. 3D porous crystalline polyimide covalent organic frameworks for drug delivery. J. Am. Chem. Soc. 137, 8352-8355 (2015).

71. Yahiaoui, O. et al. 3D anionic silicate covalent organic framework with srs topology. J. Am. Chem. Soc. 140, 5330-5333 (2018). First reported 3D COF based on non-tetrahedral building blocks.

72. Bunck, D. N. \& Dichtel, W. R. Internal functionalization of three-dimensional covalent organic frameworks. Angew. Chem. Int. Ed. 51, 1885-1889 (2012).

73. Bunck, D. N. \& Dichtel, W. R. Postsynthetic functionalization of 3D covalent organic frameworks. Chem. Commun. 49, 2457-2459 (2013). First demonstration of 3D COF post-synthetic modification.

74. Brucks, S. D., Bunck, D. N. \& Dichtel, W. R. Functionalization of 3D covalent organic frameworks using monofunctional boronic acids. Polym. (Guildf.). 55, 330-334 (2014).

75. Lu, Q. et al. Postsynthetic functionalization of three-dimensional covalent organic frameworks for selective extraction of lanthanide ions. Angew. Chem. 130, 6150-6156 (2018).

76. Huang, N., Krishna, R. \& Jiang, D. Tailor-made pore surface engineering in covalent organic frameworks: systematic functionalization for performance screening. J. Am. Chem. Soc. 137, 7079-7082 (2015).

77. Nagai, A. et al. Pore surface engineering in covalent organic frameworks. Nat. Commun. 2, 536 (2011).

78. Huang, N., Chen, X., Krishna, R. \& Jiang, D. Two-dimensional covalent organic frameworks for carbon dioxide capture through channel-wall functionalization. Angew. Chem. Int. Ed. 54, 2986-2990 (2015).

79. Chen, X. et al. Locking covalent organic frameworks with hydrogen bonds: General and remarkable effects on crystalline structure, physical properties, and photochemical activity. J. Am. Chem. Soc. 137, 3241-3247 (2015).

80. Zhang, J. et al. A novel azobenzene covalent organic framework. CrystEngComm 16, 6547-6551 (2014).

81. Deshpande, R. K., Waterhouse, G. I. N., Jameson, G. B. \& Telfer, S. G. Photolabile protecting groups in metal-organic frameworks: preventing interpenetration and masking functional groups. Chem. Commun. 48, 1574-1576 (2012).

82. Waller, P. J., Gándara, F. \& Yaghi, O. M. Chemistry of covalent organic frameworks. Acc. Chem. Res. 48, 3053-3063 (2015).

83. Smith, B. J., Overholts, A. C., Hwang, N. \& Dichtel, W. R. Insight into the crystallization of amorphous imine-linked polymer networks to $2 \mathrm{D}$ covalent organic frameworks. Chem. Commun. 52, 3690-3693 (2016).
84. Matsumoto, M. et al. Rapid, low temperature formation of imine-linked covalent organic frameworks catalyzed by metal triflates. J. Am. Chem. Soc. 139, 4999-5002 (2017).

85. Giuseppone, N., Schmitt, J. -L., Schwartz, E. \& Lehn, J. -M. Scandium(III) catalysis of transimination reactions. independent and constitutionally coupled reversible processes. J. Am. Chem. Soc. 127, 5528-5539 (2005).

86. Evans, A. M. et al. Seeded growth of single-crystal two-dimensional covalent organic frameworks. Science 361, 52-57 (2018).

87. Yuan, Y. -C., Sun, B., Cao, A. -M., Wang, D. \& Wan, L. -J. Heterogeneous nucleation and growth of highly crystalline imine-linked covalent organic frameworks. Chem. Commun. 54, 5976-5979 (2018).

88. Matsumoto, M. et al. Lewis-acid-catalyzed interfacial polymerization of covalent organic framework films. Chem 4, 308-317 (2018).

89. Dey, K. et al. Selective molecular separation by interfacially crystallized covalent organic framework thin films. J. Am. Chem. Soc. 139, 13083-13091 (2017).

90. Li, Y. et al. Growth of high-quality covalent organic framework nanosheets at the interface of two miscible organic solvents. Nanoscale Horiz. 3, 205-212 (2018).

91. Spitler, E. L. \& Dichtel, W. R. Lewis acid-catalysed formation of twodimensional phthalocyanine covalent organic frameworks. Nat. Chem. 2, 672-677 (2010).

92. Wei, T., Furgal, J. C. \& Scott, T. F. In situ deprotection and dynamic covalent assembly using a dual role catalyst. Chem. Commun. 53, 3874-3877 (2017).

93. Li, H. et al. Nucleation and growth of covalent organic frameworks from solution: the example of COF-5. J. Am. Chem. Soc. 139, 16310-16318 (2017)

94. Öhrström, L. Let's talk about MOFs-topology and terminology of metalorganic frameworks and why we need them. Crystals 5, 154-162 (2015).

95. Prasad, T. K. \& Suh, M. P. Control of interpenetration and gas-sorption properties of metal-organic frameworks by a simple change in ligand design. Chem. Eur. J. 18, 8673-8680 (2012).

96. Zhang, J., Wojtas, L., Larsen, R. W., Eddaoudi, M. \& Zaworotko, M. J. Temperature and concentration control over interpenetration in a metal -organic material. J. Am. Chem. Soc. 131, 17040-17041 (2009).

97. Farha, O. K., Malliakas, C. D., Kanatzidis, M. G. \& Hupp, J. T. Control over catenation in metal-organic frameworks via rational design of the organic building block. J. Am. Chem. Soc. 132, 950-952 (2010).

\section{Acknowledgements}

We acknowledge support from the National Science Foundation (NSF) under Award \# 1462267. X.J.M. acknowledges Rackham Graduate Student Research Grant support from the University of Michigan.

\section{Author contributions}

X.J.M. conducted the literature search, and both X.J.M. and T.F.S. contributed to writing and editing the manuscript.

\section{Additional information}

Competing interests: The authors declare no competing interests.

Reprints and permission information is available online at http://npg.nature.com/ reprintsandpermissions/

Publisher's note: Springer Nature remains neutral with regard to jurisdictional claims in published maps and institutional affiliations.

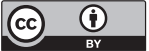

Open Access This article is licensed under a Creative Commons Attribution 4.0 International License, which permits use, sharing, adaptation, distribution and reproduction in any medium or format, as long as you give appropriate credit to the original author(s) and the source, provide a link to the Creative Commons license, and indicate if changes were made. The images or other third party material in this article are included in the article's Creative Commons license, unless indicated otherwise in a credit line to the material. If material is not included in the article's Creative Commons license and your intended use is not permitted by statutory regulation or exceeds the permitted use, you will need to obtain permission directly from the copyright holder. To view a copy of this license, visit http://creativecommons.org/ licenses/by/4.0/

(c) The Author(s) 2018 